\title{
Variants of finite full transformation semigroups
}

\author{
Igor Dolinka* \\ Department of Mathematics and Informatics \\ University of Novi Sad, Trg Dositeja Obradovića 4, 21101 Novi Sad, Serbia \\ dockie@dmi.uns.ac.rs \\ James East \\ Centre for Research in Mathematics; School of Computing, Engineering and Mathematics \\ University of Western Sydney, Locked Bag 1797, Penrith NSW 2751, Australia \\ J.East @ uws. edu . au
}

\begin{abstract}
The variant of a semigroup $S$ with respect to an element $a \in S$, denoted $S^{a}$, is the semigroup with underlying set $S$ and operation $\star$ defined by $x \star y=x a y$ for $x, y \in S$. In this article, we study variants $\mathcal{T}_{X}^{a}$ of the full transformation semigroup $\mathcal{T}_{X}$ on a finite set $X$. We explore the structure of $\mathcal{T}_{X}^{a}$ as well as its subsemigroups $\operatorname{Reg}\left(\mathcal{T}_{X}^{a}\right)$ (consisting of all regular elements) and $\mathcal{E}_{X}^{a}$ (consisting of all products of idempotents), and the ideals of $\operatorname{Reg}\left(\mathcal{T}_{X}^{a}\right)$. Among other results, we calculate the rank and idempotent rank (if applicable) of each semigroup, and (where possible) the number of (idempotent) generating sets of the minimal possible size.

Keywords: Transformation semigroups, variants, idempotents, generators, rank, idempotent rank.

MSC: 20M20; 20M10; 20M17.
\end{abstract}

\section{Introduction}

In John Howie's famous 1966 paper [39], it was shown that the semigroup $\operatorname{Sing}_{X}$ of all singular transformations on a finite set $X$ (i.e., all non-invertible functions $X \rightarrow X$ ) is generated by it idempotents. In subsequent works, and with other authors, Howie calculated the rank (minimal size of a generating set) and idempotent rank (minimal size of an idempotent generating set) of $\operatorname{Sing}_{X}$ [27, 41]; classified the idempotent generating sets of $\operatorname{Sing}_{X}$ of minimal size [41]; calculated the rank and idempotent rank of the ideals of $\operatorname{Sing}_{X}$ [44]; investigated the length function on $\operatorname{Sing}_{X}$ with respect to the generating set consisting of all idempotents of defect 1 [43]; and extended these results to various other kinds of transformation semigroups and generating sets $6,7,27,28,20]$. These works have been enormously influential, and have led to the development of several vibrant areas of research covering semigroups of (partial) transformations, matrices, partitions, endomorphisms of various algebraic structures, and more; see for example [5, $16,19,21,23,29,30,54,64]$ and references therein. The current article continues in the spirit of this program of research, but takes it in a different direction; rather than concentrating on semigroups whose elements are variations of transformations of a set, we investigate semigroups of transformations under natural alternative binary operations, studying the so-called variants of the full transformation semigroup.

The study of semigroup variants goes back to the 1960 monograph of Lyapin 50 and a 1967 paper of Magill [53] that considers semigroups of functions $X \rightarrow Y$ under an operation defined by $f \cdot g=f \circ \theta \circ g$, where $\theta$ is some fixed function $Y \rightarrow X$; see also $9,10,14,46,51,52,65,68$. In the case that $X=Y$, this provides an alternative product on the full transformation semigroup $\mathcal{T}_{X}$ (consisting of all functions $X \rightarrow X$ ) that we will have more to say about below. More generally, the variant of a semigroup $S$ with

${ }^{*}$ The first named author gratefully acknowledges the support of Grant No. 174019 of the Ministry of Education, Science, and Technological Development of the Republic of Serbia, and Grant No. 1136/2014 of the Secretariat of Science and Technological Development of the Autonomous Province of Vojvodina. 
respect to an element $a \in S$ is the semigroup, denoted $S^{a}$, with underlying set $S$ and operation $\star$ defined by $x \star y=x a y$ for each $x, y \in S$. Variants of arbitrary semigroups were first studied in 1983 by Hickey [32], where (among other things) they were used to provide a novel characterisation of Nambooripad's celebrated partial order [59] on a regular semigroup; see also [33]. As noted by Khan and Lawson [47], variants arise naturally in relation to Rees matrix semigroups, and also provide a useful alternative to the group of units in some classes of non-monoidal regular semigroups (we explore the latter idea in Section 3 below).

If $S$ is a group, it is easy to see that $S^{a}$ is isomorphic to $S$, the identity element of $S^{a}$ being $a^{-1}$; in a sense, this shows that no element of a group is more special than another, as the product may be "scaled" so that any element may play the role of the identity. When $S$ is not a group, the situation can be very different. Indeed, many semigroups with a relatively simple structure give rise to exceedingly complex variants; compare for example the right-most semigroup pictured in Figure 1 with some of its variants pictured in Figures 2 and 3 (these figures are explained in detail below) 1 In complete contrast to the situation with groups, where every variant is isomorphic to the group itself, there exist semigroups for which all the variants are pairwise non-isomorphic; the bicyclic monoid is such a semigroup [69], and some more examples may be found in [25]. On the other hand, some semigroups are isomorphic to all their variants (rectuangular bands, for example, which satisfy the identity $x a y=x y$ ).

Variants of finite full transformation semigroups have been studied in a variety of contexts. For example, Tsyaputa classified the non-isomorphic variants [70], characterised Green's relations [69] and, together with Mazorchuk, classified the isolated subsemigroups [55]; see also [49,71,72] where similar problems were considered in the context of partial transformations and partial permutations, and also 10,51,68 where more general semigroups of functions and relations are considered. The recent monograph of Ganyushkin and Mazorchuk 25] contains an entire chapter devoted to variants of various kinds of transformation semigroups, covering mostly Green's relations and the classification and enumeration of distinct variants. In the current article, we take these existing results as our point of departure, and we investigate the kind of problems discussed in the opening paragraph in the context of the variants $\mathcal{T}_{X}^{a}$ of a finite full transformation semigroup $\mathcal{T}_{X}$. The structure and main results of the article are as follows. In Sections 2 and 3 , we recall various facts regarding transformation semigroups and general variants (respectively), and also give a new characterisation of Green's relations on arbitrary variants (Proposition 3.2); from these, we deduce Tsyaputa's above-mentioned results as corollaries in Section 4 (Theorem 4.2), where we also explore the Green's structure of $\mathcal{T}_{X}^{a}$ further by investigating the natural partial order on the $\mathscr{D}$-classes, using results regarding maximal $\mathscr{D}$-classes to calculate the rank of $\mathcal{T}_{X}^{a}$ (Theorem 4.6). The most substantial part of the article constitutes an investigation, in Section 5, of the structure of $\operatorname{Reg}\left(\mathcal{T}_{X}^{a}\right)$, the subsemigroup of $\mathcal{T}_{X}^{a}$ consisting of all regular elements (the elements of $\operatorname{Reg}\left(\mathcal{T}_{X}^{a}\right)$ are characterised in Section 4, Proposition 4.1). In particular, we identify $\operatorname{Reg}\left(\mathcal{T}_{X}^{a}\right)$ as a pullback product of the regular subsemigroups of two well-known semigroups consisting of transformations with restricted range and kernel (Propositions 5.4 and 5.5), and we also show that $\operatorname{Reg}\left(\mathcal{T}_{X}^{a}\right)$ is a kind of "inflation" of the full transformation semigroup $\mathcal{T}_{A}$, where $A$ denotes the image of $a$ (Theorem 5.7); among other things, these structural results allow us to calculate the size and rank of $\operatorname{Reg}\left(\mathcal{T}_{X}^{a}\right)$ (Corollary 5.9 and Theorem 5.18). The idempotent generated subsemigroup $\mathcal{E}_{X}^{a}$ of $\mathcal{T}_{X}^{a}$ is studied in Section 6, where we characterise the elements of $\mathcal{E}_{X}^{a}$ (Theorem 6.4), calculate the rank and idempotent rank of $\mathcal{E}_{X}^{a}$ (showing in particular that these are equal, Theorem 6.8), and classify and enumerate the minimal idempotent generating sets (Theorem 6.9). Finally, in Section 7, we investigate the proper ideals of $\operatorname{Reg}\left(\mathcal{T}_{X}^{a}\right)$, showing that they are idempotent generated and calculating their rank and idempotent rank (which are again equal, Theorem 7.4).

\section{Transformation semigroups}

In this section, we record some basic notation and facts concerning finite transformation semigroups that we will need in what follows.

If $S$ is any semigroup and $U \subseteq S$, we denote by $E(U)=\left\{x \in U: x^{2}=x\right\}$ the set of all idempotents from $U$. If $U \subseteq S$, we write $\langle U\rangle$ for the subsemigroup of $S$ generated by $U$, which consists of all products $u_{1} \cdots u_{k}$ where $k \geq 1$ and $u_{1}, \ldots, u_{k} \in U$. We write $\operatorname{rank}(S)$ for the rank of $S$, defined to be the least cardinality of a

\footnotetext{
${ }^{1}$ The authors are grateful to Attila Egri-Nagy for producing the GAP code for computing with semigroup variants.
} 
subset $U \subseteq S$ such that $S=\langle U\rangle$. If $S$ is idempotent generated, we write idrank $(S)$ for the idempotent rank of $S$, defined to be the least cardinality of a subset $U \subseteq E(S)$ such that $S=\langle U\rangle$. Generation will always be in the variety of semigroups.

Recall that Green's relations $\mathscr{R}, \mathscr{L}, \mathscr{J}, \mathscr{H}, \mathscr{D}$, on a semigroup $S$ are defined, for $x, y \in S$, by

$$
\begin{gathered}
x \mathscr{R} y \Longleftrightarrow x S^{(1)}=y S^{(1)}, \quad x \mathscr{L} y \Longleftrightarrow S^{(1)} x=S^{(1)} y, \quad x \mathscr{J} y \Longleftrightarrow S^{(1)} x S^{(1)}=S^{(1)} y S^{(1)}, \\
\mathscr{H}=\mathscr{R} \cap \mathscr{L}, \quad \mathscr{D}=\mathscr{R} \circ \mathscr{L}=\mathscr{L} \circ \mathscr{R} .
\end{gathered}
$$

Here, $S^{(1)}$ denotes the monoid obtained from $S$ by adjoining an identity element 1 , if necessary. (We use the notation $S^{(1)}$ rather than the more standard $S^{1}$ for reasons that will become clear shortly.) If $x \in S$, and if $\mathscr{K}$ is one of $\mathscr{R}, \mathscr{L}, \mathscr{J}, \mathscr{H}, \mathscr{D}$, we denote by $K_{x}$ the $\mathscr{K}$-class of $x$ in $S$. An $\mathscr{H}$-class contains an idempotent if and only if it is a group, in which case it is a maximal subgroup of $S$. The $\mathscr{J}$-classes of $S$ are partially ordered; we say that $J_{x} \leq J_{y}$ if $x \in S^{(1)} y S^{(1)}$. If $S$ is finite, then $\mathscr{J}=\mathscr{D}$. An element $x \in S$ is regular if $x=x y x$ and $y=y x y$ for some $y \in S$ or, equivalently, if $D_{x}$ contains an idempotent, in which case $R_{x}$ and $L_{x}$ do, too. We write $\operatorname{Reg}(S)$ for the set of all regular elements of $S$, and we say $S$ is regular if $S=\operatorname{Reg}(S)$.

Let $X$ be a finite set with $|X|=n$. The full transformation semigroup on $X$ is the (regular) semigroup $\mathcal{T}_{X}$ of all transformations of $X$ (i.e., all functions $X \rightarrow X$ ), under the operation of composition. We write $x f$ for the image of $x \in X$ under $f \in \mathcal{T}_{X}$, and we compose functions from left to right. If $f \in \mathcal{T}_{X}$, we will write

$$
f=\left(\begin{array}{ccc}
F_{1} & \cdots & F_{m} \\
f_{1} & \cdots & f_{m}
\end{array}\right)
$$

to indicate that $X=F_{1} \sqcup \cdots \sqcup F_{m}$ and $F_{i} f=f_{i}$ for each $i$. (The symbol "ப" denotes disjoint union.) Usually this notation will imply that $f_{1}, \ldots, f_{m}$ are distinct, but occasionally this will not be the case, and we will always specify this. As usual, we denote the image, kernel and rank of $f \in \mathcal{T}_{X}$ by

$$
\operatorname{im}(f)=\{x f: x \in X\}, \quad \operatorname{ker}(f)=\{(x, y) \in X \times X: x f=y f\}, \quad \operatorname{rank}(f)=|\operatorname{im}(f)|=|X / \operatorname{ker}(f)| .
$$

We will sometimes write $\operatorname{ker}(f)=\left(F_{1}|\cdots| F_{m}\right)$ to indicate that $\operatorname{ker}(f)$ has equivalence classes $F_{1}, \ldots, F_{m}$, and this notation will always imply that the $F_{i}$ are pairwise disjoint and non-empty. The symmetric group on $X$ is the set $\mathcal{S}_{X}$ of all permutations of $X$ (i.e., all invertible functions $X \rightarrow X$ ) and is the group of units of $\mathcal{T}_{X}$. In the case that $X=\{1, \ldots, n\}$, we will write $\mathcal{T}_{X}=\mathcal{T}_{n}$ and $\mathcal{S}_{X}=\mathcal{S}_{n}$. In general, if $k$ is a non-negative integer, we will write $\mathbf{k}=\{1, \ldots, k\}$. (So $\mathbf{k}=\emptyset$ if $k=0$.) A transformation $f \in \mathcal{T}_{n}$ will often be written as $f=[1 f, \ldots, n f]$. Green's relations on $\mathcal{T}_{X}$ are easy to describe; see for example [37,42.

Proposition 2.1. If $f \in \mathcal{T}_{X}$, where $X$ is a finite set with $|X|=n$, then

(i) $R_{f}=\left\{g \in \mathcal{T}_{X}: \operatorname{ker}(f)=\operatorname{ker}(g)\right\}$,

(ii) $L_{f}=\left\{g \in \mathcal{T}_{X}: \operatorname{im}(f)=\operatorname{im}(g)\right\}$,

(iii) $H_{f}=\left\{g \in \mathcal{T}_{X}: \operatorname{ker}(f)=\operatorname{ker}(g)\right.$ and $\left.\operatorname{im}(f)=\operatorname{im}(g)\right\}$,

(iv) $D_{f}=\left\{g \in \mathcal{T}_{X}: \operatorname{rank}(f)=\operatorname{rank}(g)\right\}$.

The $\mathscr{D}$-classes of $\mathcal{T}_{X}$ form a chain: $D_{1}<\cdots<D_{n}$, where $D_{m}=\left\{f \in \mathcal{T}_{X}: \operatorname{rank}(f)=m\right\}$ for each $m \in \mathbf{n}$. A group $\mathscr{H}$-class contained in $D_{m}$ is isomorphic to $\mathcal{S}_{m}$.

Note that $D_{n}=\mathcal{S}_{X}$. For future reference, Figure 1 gives the so-called egg box diagrams of the semigroups $\mathcal{T}_{1}, \mathcal{T}_{2}, \mathcal{T}_{3}, \mathcal{T}_{4}$. Large boxes are $\mathscr{D}$-classes; within a $\mathscr{D}$-class, $\mathscr{R}$-related (resp., $\mathscr{L}$-related) elements are in the same row (resp., column); $\mathscr{H}$-related elements are in the same cell; group $\mathscr{H}$-classes are shaded grey and the label "m" indicates that a given group is isomorphic to $\mathcal{S}_{m}$; the $\mathscr{J}=\mathscr{D}$-order is indicated by the edges between $\mathscr{D}$-classes. (See 37,42$]$ for more on egg box diagrams.) The pictures were produced with the SEMIGROUPS package [57] on GAP [26].

It is well-known that $\operatorname{rank}\left(\mathcal{S}_{X}\right)=2$ and $\operatorname{rank}\left(\mathcal{T}_{X}\right)=3$ if $|X| \geq 3$; for example, $\mathcal{S}_{n}$ is generated by the transposition $[2,1,3,4, \ldots, n]$ and $n$-cycle $[2,3,4, \ldots, n, 1]$, while $\mathcal{T}_{X}$ is generated by (any generating set 


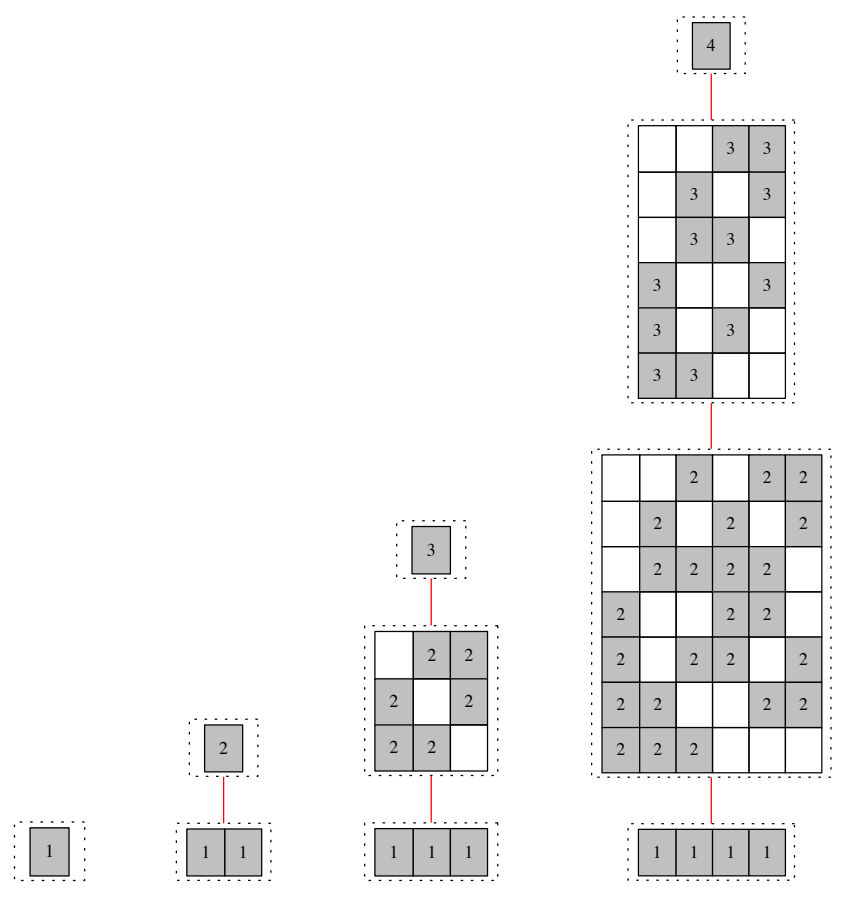

Figure 1: Egg box diagrams of the semigroups $\mathcal{T}_{1}, \mathcal{T}_{2}, \mathcal{T}_{3}, \mathcal{T}_{4}$ (left to right).

for) $\mathcal{S}_{X}$ along with any element of $D_{n-1}$; see for example [1, 25, 58, 73]. The set $E\left(\mathcal{T}_{X}\right)$ of idempotents of $\mathcal{T}_{X}$ is not a subsemigroup, but the idempotent generated subsemigroup $\mathcal{E}_{X}=\left\langle E\left(\mathcal{T}_{X}\right)\right\rangle$ of $\mathcal{T}_{X}$ has a neat description. For $x, y \in X$ with $x \neq y$, denote by $\varepsilon_{x y}$ the (idempotent) transformation defined, for $z \in X$, by

$$
z \varepsilon_{x y}= \begin{cases}x & \text { if } z=y \\ z & \text { if } z \neq y\end{cases}
$$

Then $E\left(D_{n-1}\right)=\left\{\varepsilon_{x y}: x, y \in X, x \neq y\right\}$.

Theorem 2.2 (Howie [39, 41]; Gomes and Howie [27]). If $X$ is a finite set with $|X|=n \geq 2$, then

$$
\mathcal{E}_{X}=\left\langle E\left(\mathcal{T}_{X}\right)\right\rangle=\{1\} \cup\left(\mathcal{T}_{X} \backslash \mathcal{S}_{X}\right) \quad \text { and } \quad\left\langle E\left(D_{n-1}\right)\right\rangle=\mathcal{T}_{X} \backslash \mathcal{S}_{X} .
$$

Further, $\operatorname{rank}\left(\mathcal{T}_{X} \backslash \mathcal{S}_{X}\right)=\operatorname{idrank}\left(\mathcal{T}_{X} \backslash \mathcal{S}_{X}\right)=\rho_{n}$, where $\rho_{2}=2$ and $\rho_{n}=\left(\begin{array}{l}n \\ 2\end{array}\right)$ if $n \geq 3$.

The minimal idempotent generating sets of $\mathcal{T}_{X} \backslash \mathcal{S}_{X}$ have a nice graphical interpretation. Recall that a tournament on $X$ is a directed graph $\Gamma$ with vertex set $X$ such that for each $x, y \in X$ with $x \neq y, \Gamma$ contains precisely one of the directed edges $(x, y)$ or $(y, x)$. Recall also that a directed graph on vertex set $X$ is strongly connected if for any $x, y \in X$, there is a directed path from $x$ to $y$ in $\Gamma$. If $|X| \geq 3$, we will write $\mathbb{T}_{X}$ for the set of all strongly connected tournaments on $X$. By convention, if $X=\{x, y\}$ is a set of size 2 , we will let $\mathbb{T}_{X}$ denote the set consisting of a single graph; namely, the graph with vertex set $X$ and directed edges $(x, y)$ and $(y, x)$. For $U \subseteq E\left(D_{n-1}\right)$, we define a graph $\Gamma_{U}$ on vertex set $X$ with a directed edge $(x, y)$ corresponding to each $\varepsilon_{x y} \in U$.

Theorem 2.3 (Howie [41]). Let $X$ be a finite set with $|X|=n \geq 2$, and let

$$
U \subseteq E\left(D_{n-1}\right)=\left\{\varepsilon_{x y}: x, y \in X, x \neq y\right\}
$$

with $|U|=\rho_{n}$ (as defined in Theorem 2.2). Then $\mathcal{T}_{X} \backslash \mathcal{S}_{X}=\langle U\rangle$ if and only if $\Gamma_{U} \in \mathbb{T}_{X}$. In particular, the number of idempotent generating sets of the minimal size $\rho_{n}$ is equal to $\left|\mathbb{T}_{X}\right|$.

Remark 2.4. A recurrence relation for the numbers $\left|\mathbb{T}_{X}\right|$ is given in $[74]$. The current authors have shown 15 that any idempotent generating set for $\mathcal{T}_{X} \backslash \mathcal{S}_{X}$ contains one of minimal possible size; a formula was also given for the total number of subsets of $E\left(D_{n-1}\right)$ that generate $\mathcal{T}_{X} \backslash \mathcal{S}_{X}$ (but are not necessarily of size $\rho_{n}$ ). Arbitrary generating sets of minimal size were classified in [5]. The subsemigroup generated by the idempotents of an infinite transformation semigroup was described in 39 . 


\section{$3 \quad$ Variant semigroups}

Let $S$ be a semigroup, and fix some element $a \in S$. A new operation $\star_{a}$ may be defined on $S$ by

$$
x \star_{a} y=x a y \quad \text { for each } x, y \in S .
$$

We write $S^{a}$ for the semigroup $\left(S, \star_{a}\right)$ obtained in this fashion, and call $S^{a}$ the variant of $S$ with respect to $a$. Since we fix $S$ and $a$ throughout this section, we will supress the subscript and simply write $\star$ for $\star_{a}$. (Note that several authors write $\circ_{a}$ instead of $\star_{a}$, but we use the current notation so as not to interfere with the usual use of $\circ$ to denote composition of functions in $\mathcal{T}_{X}$.)

If $u, v \in S^{(1)}$, the map $x \mapsto v x u$ defines a homomorphism $S^{u a v} \rightarrow S^{a}$. If $S$ is a monoid with identity 1 , we write $G(S)$ for the group of units of $S$; that is,

$$
G(S)=\{x \in S:(\exists y \in S) x y=y x=1\} .
$$

We have already noted that $G\left(\mathcal{T}_{X}\right)=\mathcal{S}_{X}$. If $S$ is a monoid and $u, v \in G(S)$ are units, then the above map $S^{\text {uav }} \rightarrow S^{a}$ is invertible and, hence, an isomorphism. As a special case, if $a$ is a unit, the maps $x \mapsto x a$ and $x \mapsto a x$ define isomorphisms $S^{a} \rightarrow S=S^{1}$. As a result, we will typically concern ourselves only with the case that $a$ is not a unit (although $S$ may in fact be a monoid), and call $S^{a}$ a non-trivial variant in this case. Our main objects of study are the (non-trivial) variants of a finite full transformation semigroup $\mathcal{T}_{X}$, but in this section we will prove some general results concerning arbitrary variants.

Before we do this, it is instructive to consider some examples; Figures 2 and 3 illustrate the egg box diagrams of the variant semigroup $\mathcal{T}_{4}^{a}$ for various choices of $a \in \mathcal{T}_{4}$. A number of things become apparent when

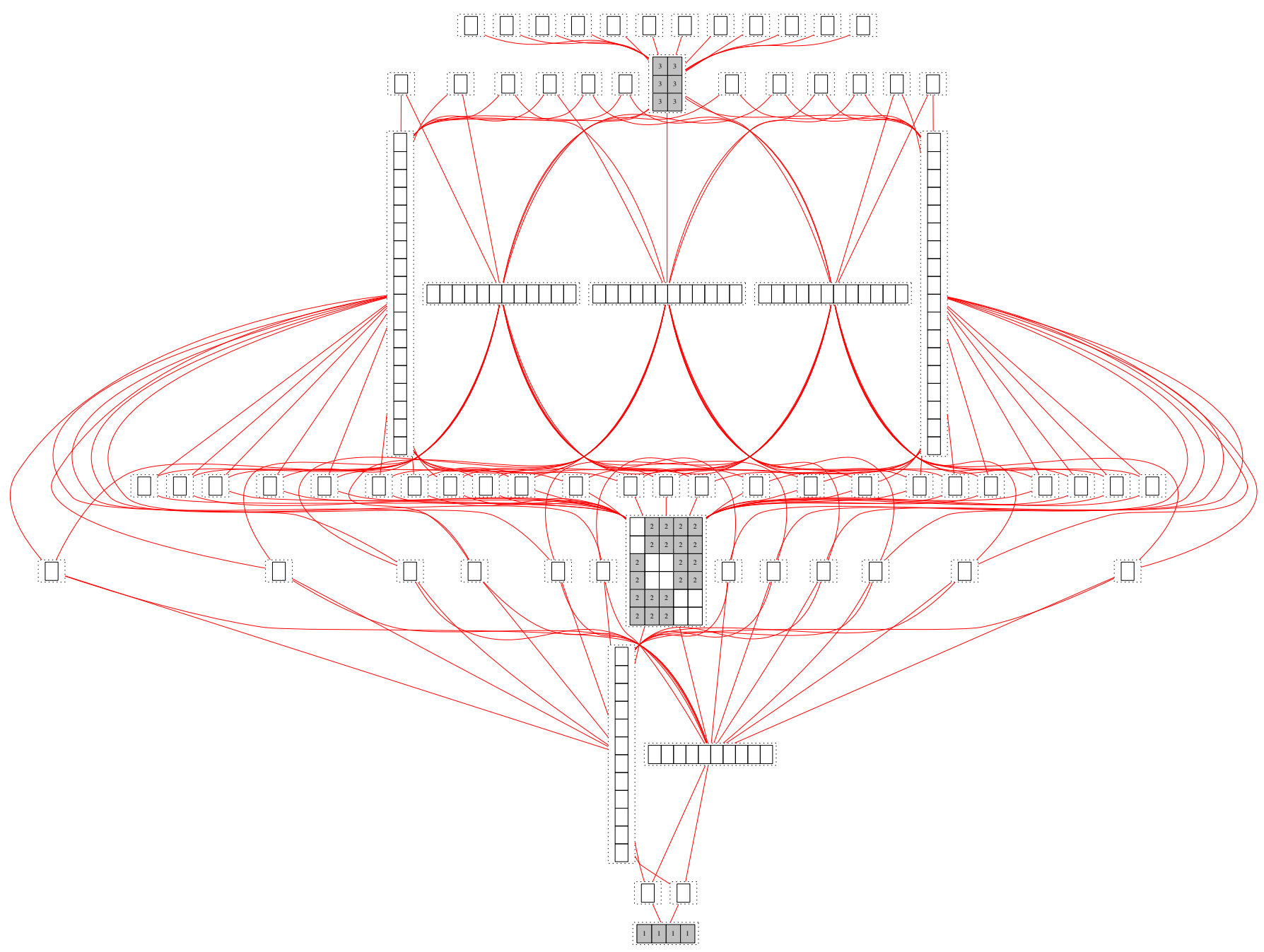

Figure 2: Egg box diagram of the variant semigroup $\mathcal{T}_{4}^{a}$, where $a=[1,2,3,3]$.

examining Figures 2 and 3. In each case: 


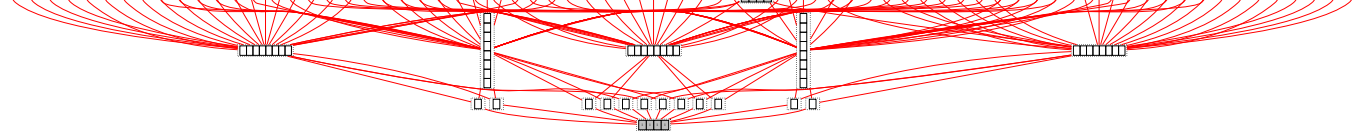

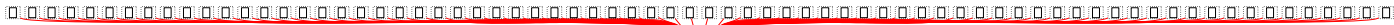

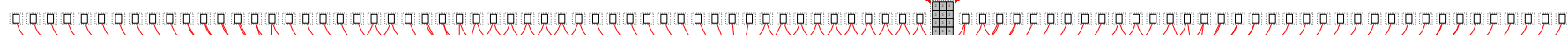

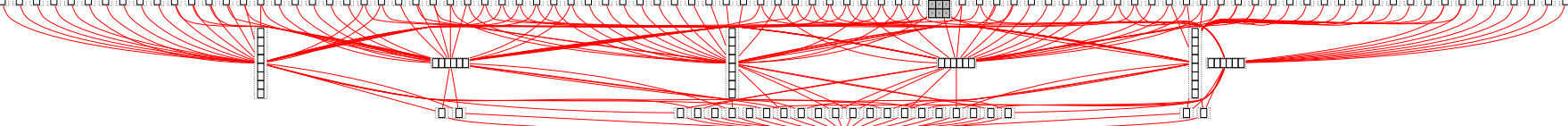

Figure 3: Egg box diagrams of the variant semigroups $\mathcal{T}_{4}^{a}$, where $a=[1,1,2,2]$ (top) and $a=[1,2,2,2]$ (bottom). The pdf file may be zoomed to obtain greater clarity.

(i) $\mathcal{T}_{4}^{a}$ is not regular (as indicated by the many $\mathscr{D}$-classes containing no idempotents).

(ii) A non-regular $\mathscr{D}$-class of $\mathcal{T}_{4}^{a}$ is either a single $\mathscr{R}$-class or a single $\mathscr{L}$-class, or both (so a single $\mathscr{H}$-class).

(iii) All the maximal $\mathscr{D}$-classes are single $\mathscr{H}$-classes (but a $\mathscr{D}$-class consisting of a single $\mathscr{H}$-class need not be maximal).

(iv) The number of maximal $\mathscr{D}$-classes increases as $r=\operatorname{rank}(a)$ decreases.

(v) It is not evident from the picture, but every $\mathscr{H}$-class contained in a non-regular $\mathscr{D}$-class is a singleton.

In fact, all of these statements are true for arbitrary non-trivial variants of a finite transformation semigroup, while some are true for variants of arbitrary semigroups, as we will soon see.

We now prove a result concerning Green's relations on $S^{a}$. In order to avoid confusion, if $\mathscr{K}$ is one of $\mathscr{R}$, $\mathscr{L}, \mathscr{J}, \mathscr{H}, \mathscr{D}$, we will write $\mathscr{K}^{a}$ for Green's $\mathscr{K}$-relation on the variant $S^{a}$, and write $K_{x}^{a}$ for the $\mathscr{K}^{a}$-class of $x \in S^{a}$. It is easy to check that $\mathscr{K}^{a} \subseteq \mathscr{K}$ for each relation $\mathscr{K}$ and, hence, $K_{x}^{a} \subseteq K_{x}$ for each $x \in S$. Throughout our investigations, a crucial role will be played by the sets

$$
P_{1}=\{x \in S: x a \mathscr{R} x\}, \quad P_{2}=\{x \in S: a x \mathscr{L} x\}, \quad P=P_{1} \cap P_{2} .
$$

We note that $P_{1}=P_{2}=P=S$ if $S$ is a monoid and $a \in G(S)$ is a unit.

Lemma 3.1. If $y \in S$, then $\beta$

(i) $y \in P_{1}$ if and only if $L_{y} \subseteq P_{1}$,

(ii) $y \in P_{2}$ if and only if $R_{y} \subseteq P_{2}$.

The set $\operatorname{Reg}\left(S^{a}\right)$ of all regular elements of $S^{a}$ is contained in $P=P_{1} \cap P_{2}$.

Proof. We just prove (i) because (ii) is dual. Suppose $y \in P_{1}$, and let $z \in L_{y}$ be arbitrary. So y $\mathscr{R}$ ya, and we have $z=u y$ for some $u \in S^{(1)}$. But then $z=u y \mathscr{R} u y a=z a$ since $\mathscr{R}$ is a left congruence, so $z \in P_{1}$, whence $L_{y} \subseteq P_{1}$. The other implication is trivial. For the statement about regular elements, note that if $x \in \operatorname{Reg}\left(S^{a}\right)$, then $x=x \star y \star x=x a y a x$ for some $y \in S$. This gives $x a \mathscr{R} x \mathscr{L}$ ax, so $x \in P$.

Proposition 3.2. If $x \in S$, then
(i) $R_{x}^{a}= \begin{cases}R_{x} \cap P_{1} & \text { if } x \in P_{1} \\ \{x\} & \text { if } x \in S \backslash P_{1},\end{cases}$
(iii) $H_{x}^{a}= \begin{cases}H_{x} & \text { if } x \in P \\ \{x\} & \text { if } x \in S \backslash P,\end{cases}$
(ii) $L_{x}^{a}= \begin{cases}L_{x} \cap P_{2} & \text { if } x \in P_{2} \\ \{x\} & \text { if } x \in S \backslash P_{2},\end{cases}$
(iv) $D_{x}^{a}= \begin{cases}D_{x} \cap P & \text { if } x \in P \\ L_{x}^{a} & \text { if } x \in P_{2} \backslash P_{1} \\ R_{x}^{a} & \text { if } x \in P_{1} \backslash P_{2} \\ \{x\} & \text { if } x \in S \backslash\left(P_{1} \cup P_{2}\right) .\end{cases}$ 
Further, if $x \in S \backslash P$, then $H_{x}^{a}=\{x\}$ is a non-group $\mathscr{H}^{a}$-class of $S^{a}$.

Proof. We begin with (i). Suppose $y \in R_{x}^{a} \backslash\{x\}$. Then $x=y \star u=y a u$ and $y=x \star v=x a v$ for some $u, v \in S$. But then $x=y a u=x a(v a u)$, so that $x \mathscr{R} x a$, and $x \in P_{1}$. In particular, if $x \in S \backslash P_{1}$, then $R_{x}^{a}=\{x\}$. Next, suppose $x \in P_{1}$. If $y$ is another element of $R_{x}^{a}$ then, since $R_{y}^{a}=R_{x}^{a}$, the previous calculation shows that $y \in P_{1}$, and it follows that $R_{x}^{a} \subseteq P_{1}$. Since we have already observed that $R_{x}^{a} \subseteq R_{x}$, it follows that $R_{x}^{a} \subseteq R_{x} \cap P_{1}$. Conversely, suppose $y \in R_{x} \cap P_{1}$. If $y=x$, then $y \in R_{x}^{a}$, so suppose $y \neq x$. So $x=y u$ and $y=x v$ for some $u, v \in S$. Also, $x=x a w$ and $y=y a z$ for some $w, z \in S^{(1)}$, since $x, y \in P_{1}$. Then $x=y u=y a z u=y \star(z u)$ and, similarly, $y=x \star(w v)$, showing that $y \in R_{x}^{a}$.

Part (ii) is dual to (i). We now prove (iii). If $x \in S \backslash P$, then either $R_{x}^{a}=\{x\}$ or $L_{x}^{a}=\{x\}$ (or both). In any case, $H_{x}^{a}=R_{x}^{a} \cap L_{x}^{a}=\{x\}$. Next, suppose $x \in P$. We have already noted that $H_{x}^{a} \subseteq H_{x}$. Conversely, suppose $y \in H_{x}$. If $y=x$, then $y \in H_{x}^{a}$, so suppose $y \neq x$. Then $x=y s=t y$ and $y=x u=v x$ for some $s, t, u, v \in S$. Also, $x=x a w=z a x$ for some $w, z \in S^{(1)}$, since $x \in P$. But then $y=x u=x a w u=x \star(w u)$ and $x=y s=v x s=v x a w s=y a w s=y \star(w s)$, showing that $y \mathscr{R}^{a} x$. A similar calculation shows that y $\mathscr{L}^{a} x$, and we conclude that $y \in H_{x}^{a}$.

For part (iv), note that

$$
D_{x}^{a}=\bigcup_{y \in R_{x}^{a}} L_{y}^{a}=\bigcup_{y \in L_{x}^{a}} R_{y}^{a} .
$$

In particular, if $x \in S \backslash P_{1}$, then $R_{x}^{a}=\{x\}$, so that $D_{x}^{a}=L_{x}^{a}$. Similarly, if $x \in S \backslash P_{2}$, then $D_{x}^{a}=R_{x}^{a}$. If $x \in S \backslash\left(P_{1} \cup P_{2}\right)=\left(S \backslash P_{1}\right) \cap\left(S \backslash P_{2}\right)$, then $D_{x}^{a}=L_{x}^{a}=\{x\}$. Finally, if $x \in P$, then

$$
D_{x}^{a}=\bigcup_{y \in R_{x}^{a}} L_{y}^{a}=\bigcup_{y \in R_{x} \cap P_{1}}\left(L_{y} \cap P_{2}\right)=P_{2} \cap \bigcup_{y \in R_{x} \cap P_{1}} L_{y}=P_{2} \cap \bigcup_{y \in R_{x}}\left(L_{y} \cap P_{1}\right)=P \cap \bigcup_{y \in R_{x}} L_{y}=P \cap D_{x},
$$

where we have used parts (i) and (ii) in the second step, and Lemma 3.1 (which tells us that $L_{y} \cap P_{1}$ is equal to $L_{y}$ or $\emptyset$ if $y \in P_{1}$ or $y \notin P_{1}$, respectively) in the fourth step.

For the final statement about group $\mathscr{H}^{a}$-classes, suppose $H_{x}^{a}$ is a group, and let $e$ be the identity element of this group. Then $x=x \star e=x a e$ and also $x=e a x$, so it follows that $x a \mathscr{R} x \mathscr{L}$ ax, whence $x \in P$.

Remark 3.3. In a sequel to the current paper [14], we characterise the $\mathscr{J}^{a}$ relation, but we do not need this here. As noted above, if $S$ is a monoid and $a \in G(S)$ a unit, then $P_{1}=P_{2}=P=S$, in which case Green's relations on $S^{a}$ coincide exactly with the corresponding relations on $S \cong S^{a}$. Let $x \in P=P_{1} \cap P_{2}$, and put $H=H_{x}^{a}=H_{x}$. Whether $H$ is a group or non-group $\mathscr{H}$-class of $S$ is independent of whether $H$ is a group or non-group $\mathscr{H}^{a}$-class of $S^{a}$. See Table 1 for some examples with $S=\mathcal{T}_{4}, a=[1,2,3,3]$ and $x \in P$. (See the next section for a description of the set $P$ in the case of $S=\mathcal{T}_{X}$.)

\begin{tabular}{|c|c|c|}
\hline$x$ & Is $H_{x}$ a group $\mathscr{H}$-class of $\mathcal{T}_{4} ?$ & Is $H_{x}$ a group $\mathscr{H}^{a}$-class of $\mathcal{T}_{4}^{a} ?$ \\
\hline$[1,1,3,3]$ & Yes & Yes \\
{$[4,2,2,4]$} & Yes & No \\
{$[2,4,2,4]$} & No & Yes \\
{$[1,3,1,3]$} & No & No \\
\hline
\end{tabular}

Table 1: Group/non-group relationships between $H_{x}$ and $H_{x}^{a}$ in $\mathcal{T}_{4}$ and $\mathcal{T}_{4}^{a}$, where $a=[1,2,3,3]$.

If $S$ is a monoid and $a \in G(S)$, then $S^{a}$ is a monoid (since then $S^{a} \cong S$ ). The converse of this statement is also true, as we now demonstrate. Part of the next proof is similar to that of [25, Proposition 13.1.1].

Proposition 3.4. Let $S$ be a semigroup and let $a \in S$. Then $S^{a}$ is a monoid if and only if $S$ is a monoid and $a \in G(S)$, in which case $S^{a}$ is isomorphic to $S$.

Proof. It suffices to show the forwards implication, so suppose $S^{a}$ is a monoid with identity $e$. In particular, for each $x \in S, x=x \star e=e \star x$; that is, $x=x a e=e a x$ for all $x$. So ae is a right identity for $S$, and $e a$ a left identity. It follows that $a e=e a$ is a two sided identity for $S$, and that $a$ is a unit (with inverse $e$ ). 
So $S^{a}$ is not a monoid in general, even if $S$ is itself a monoid. The idea of the group of units of a monoid may be generalised to a non-unital semigroup $S$ by considering the so-called regularity presering elements of $S$ [32,47]; namely, those elements $a \in S$ for which $S^{a}$ is a regular semigroup. The set of all regularity preserving elements of $S$ is denoted $\operatorname{RP}(S)$. As the use of the word "preserving" suggests, $S$ can only contain regularity preserving elements if $S$ is itself regular, as may easily be checked (though there are regular semigroups $S$ for which $\operatorname{RP}(S)=\emptyset$, one example being $S=\mathcal{T}_{X} \backslash \mathcal{S}_{X}$ ). It is also clear that if $a \in \operatorname{RP}(S)$, then $J_{a}$ must be a maximum element in the ordering of $\mathscr{J}$-classes. If $S$ is a regular monoid, then $\operatorname{RP}(S)=G(S)$, and this is just one of the reasons that $\operatorname{RP}(S)$ is considered to be a good analogue of the group of units in the case that $S$ is not a monoid. The next result summarises some of the facts from [47] that we will need when investigating regularity preserving elements later. Recall that an element $u \in S$ is a mididentity (sometimes called a midunit or middle unit) if $x u y=x y$ for all $x, y \in S$. Semigroups with mididentity were first studied in 75$]$ (the idea is also present in [67]), and then more systematically in [3,4]; the connection with semigroup variants is elucidated in [8,32, 47].

Proposition 3.5 (Khan and Lawson [47]). Let $S$ be a regular semigroup.

(i) An element $a \in S$ is regularity preserving if and only if a $\mathscr{H}$ e for some regularity preserving idempotent $e \in E(S)$. (In particular, $\operatorname{RP}(S)$ is a union of groups.)

(ii) An idempotent $e \in E(S)$ is regularity preserving if and only if fe $\mathscr{R} f \mathscr{L}$ ef for all idempotents $f \in E(S)$.

(iii) Any mididentity is regularity preserving.

So $S^{a}$ is not regular in general, even though $S$ may be regular itself. But in some cases, $\operatorname{Reg}\left(S^{a}\right)$, the set of all regular elements of $S_{a}$, is a subsemigroup of $S$. The next result was proved in [47] under the assumption that $S$ is regular, but the proof given there works unmodified for the following stronger statement.

Lemma 3.6 (Khan and Lawson 47]). Suppose $S$ is a semigroup, and that $a S a \subseteq \operatorname{Reg}(S)$ for some a $\in$. Then $\operatorname{Reg}\left(S^{a}\right)$ is a (regular) subsemigroup of $S^{a}$.

\section{The variant semigroup $\mathcal{T}_{X}^{a}$}

We now turn our attention to the main object of our study; namely, the variants $\mathcal{T}_{X}^{a}$, where $X$ is a finite set with $|X|=n$ and $a \in \mathcal{T}_{X}$. The main results of this section include a characterisation of Green's relations and the ordering on $\mathscr{J}=\mathscr{D}$-classes, and the calculation of $\operatorname{rank}\left(\mathcal{T}_{X}^{a}\right)$.

It is easy to see that for any $a \in \mathcal{T}_{X}$, there is a permutation $p \in \mathcal{S}_{X}=G\left(\mathcal{T}_{X}\right)$ such that $a p \in E\left(\mathcal{T}_{X}\right)$ is an idempotent. As noted in the previous section, $\mathcal{T}_{X}^{a}$ and $\mathcal{T}_{X}^{a p}$ are then isomorphic, so it suffices to assume that $a$ is an idempotent. So for the remainder of the article, we fix an idempotent $a \in E\left(\mathcal{T}_{X}\right)$ with $r=\operatorname{rank}(a)$, and we write

$$
a=\left(\begin{array}{ccc}
A_{1} & \cdots & A_{r} \\
a_{1} & \cdots & a_{r}
\end{array}\right) .
$$

The condition that $a$ is an idempotent is equivalent to saying that $a_{i} \in A_{i}$ for each $i \in \mathbf{r}$. Further, we will write $A=\operatorname{im}(a)=\left\{a_{1}, \ldots, a_{r}\right\}$ and $\alpha=\operatorname{ker}(a)=\left(A_{1}|\cdots| A_{r}\right)$. We will also write $\lambda_{i}=\left|A_{i}\right|$ for each $i$, and for $I=\left\{i_{1}, \ldots, i_{m}\right\} \subseteq \mathbf{r}$, we define $\Lambda_{I}=\lambda_{i_{1}} \cdots \lambda_{i_{m}}$. In the special case that $I=\mathbf{r}$, we will write $\Lambda=\Lambda_{\mathrm{r}}=\lambda_{1} \cdots \lambda_{r}$. As in the previous section, we will write $\star$ for $\star_{a}$. If $r=n$, then $a \in \mathcal{S}_{X}=G\left(\mathcal{T}_{X}\right)$ and so, as we have noted, $\mathcal{T}_{X}^{a} \cong \mathcal{T}_{X}$. All the problems we consider have been solved for $\mathcal{T}_{X}$, so we will assume throughout that $r<n$. In particular, $\mathcal{T}_{X}^{a}$ is not a monoid, nor regular since $\mathcal{S}_{X}=\operatorname{RP}\left(\mathcal{T}_{X}\right)$.

As in the previous section, we will write $\mathscr{R}, \mathscr{L}, \mathscr{H}, \mathscr{D}=\mathscr{J}$ for Green's relations on $\mathcal{T}_{X}$, and $\mathscr{R}^{a}, \mathscr{L}^{a}, \mathscr{H}^{a}$, $\mathscr{D}^{a}=\mathscr{J}^{a}$ for Green's relations on $\mathcal{T}_{X}^{a}$. If $f \in \mathcal{T}_{X}$ and if $\mathscr{K}$ is one of $\mathscr{R}, \mathscr{L}, \mathscr{H}, \mathscr{D}$, we write $K_{f}$ and $K_{f}^{a}$ for the $\mathscr{K}$-class and $\mathscr{K}^{a}$-class of $f$, respectively. As we noted in the previous section for arbitrary variant semigroups, $\mathscr{K}^{a} \subseteq \mathscr{K}$ for each $\mathscr{K}$ and, hence, $K_{f}^{a} \subseteq K_{f}$ for each $f$. 
As we have seen, the key to describing Green's relations on $\mathcal{T}_{X}^{a}$ are the sets

$$
P_{1}=\left\{f \in \mathcal{T}_{X}: f a \mathscr{R} f\right\}, \quad P_{2}=\left\{f \in \mathcal{T}_{X}: \text { af } \mathscr{L} f\right\}, \quad P=P_{1} \cap P_{2} .
$$

It will be convenient to have a more transparent characterisation of the elements of $P_{1}$ and $P_{2}$. In order to give such a description, we introduce some terminology. Let $B$ be a subset of $X$ and $\beta$ an equivalence relation on $X$. We say $B$ saturates $\beta$ if each $\beta$-class contains at least one element of $B$. We say $\beta$ separates $B$ if each $\beta$-class contains at most one element of $B$. We call $B$ a cross-section of $\beta$ if $B$ saturates and is separated by $\beta$.

Proposition 4.1. (i) $P_{1}=\left\{f \in \mathcal{T}_{X}: \operatorname{rank}(f a)=\operatorname{rank}(f)\right\}=\left\{f \in \mathcal{T}_{X}: \alpha\right.$ separates $\left.\operatorname{im}(f)\right\}$,

(ii) $P_{2}=\left\{f \in \mathcal{T}_{X}: \operatorname{rank}(a f)=\operatorname{rank}(f)\right\}=\left\{f \in \mathcal{T}_{X}: A\right.$ saturates $\left.\operatorname{ker}(f)\right\}$,

(iii) $P=\left\{f \in \mathcal{T}_{X}: \operatorname{rank}(a f a)=\operatorname{rank}(f)\right\}=\operatorname{Reg}\left(\mathcal{T}_{X}^{a}\right)$ is the set of all regular elements of $\mathcal{T}_{X}^{a}$, and is a subsemigroup of $\mathcal{T}_{X}^{a}$.

Proof. Let $f \in \mathcal{T}_{X}$ and write $f=\left(\begin{array}{ccc}F_{1} & \cdots & F_{m} \\ f_{1} & \cdots & f_{m}\end{array}\right)$, where $m=\operatorname{rank}(f)$. For each $i \in \mathbf{m}$, let $k_{i} \in \mathbf{r}$ be such that $f_{i} \in A_{k_{i}}$. Note that

$$
f \in P_{1} \Longleftrightarrow f a \mathscr{R} f \Longleftrightarrow \operatorname{ker}(f a)=\operatorname{ker}(f) \Longleftrightarrow \operatorname{rank}(f a)=\operatorname{rank}(f),
$$

since $X$ is finite. Note that for each $i \in \mathbf{m}, F_{i} f a=f_{i} a=a_{k_{i}}$. It follows that $\operatorname{rank}(f a)=m$ if and only if the set $\left\{k_{1}, \ldots, k_{m}\right\}$ has cardinality $m$, and this is clearly equivalent to $\alpha$ separating $\operatorname{im}(f)$, establishing (i).

A similar argument shows that $f \in P_{2}$ if and only if $\operatorname{rank}(a f)=\operatorname{rank}(f)$. Next, note that $\operatorname{im}(a f) \subseteq \operatorname{im}(f)$ and that for all $i \in \mathbf{m}, f_{i}(a f)^{-1}=F_{i} a^{-1}=\bigcup_{a_{j} \in F_{i}} A_{j}$. So $\operatorname{rank}(a f)=m$ if and only if $F_{i} \cap A \neq \emptyset$ for all $i$, and this is clearly equivalent to $A$ saturating $\operatorname{ker}(f)$, giving (ii).

Combining the arguments of the previous two paragraphs shows that $f \in P=P_{1} \cap P_{2}$ if and only if $\operatorname{rank}(a f a)=\operatorname{rank}(f)$. We have already seen in Lemma 3.1 that $\operatorname{Reg}\left(\mathcal{T}_{X}^{a}\right) \subseteq P$. Conversely, suppose $f \in P$. Since $\operatorname{rank}(f a)=\operatorname{rank}(a f)=m$, we may write $f a=\left(\begin{array}{ccc}F_{1} & \ldots & F_{m} \\ a_{k_{1}} & \cdots & a_{k_{m}}\end{array}\right)$ and $a f=\left(\begin{array}{ccc}G_{1} & \cdots & G_{m} \\ f_{1} & \cdots & f_{m}\end{array}\right)$, where $k_{1}, \ldots, k_{m}$ are distinct, and $G_{1}, \ldots, G_{m}$ are non-empty and pairwise disjoint. Let $g \in \mathcal{T}_{X}$ be any transformation for which $a_{k_{i}} g \in G_{i}$ for each $i \in \mathbf{m}$. Then clearly, $f=(f a) g(a f)=f \star g \star f$, showing that $f \in \operatorname{Reg}\left(\mathcal{T}_{X}^{a}\right)$. Finally, Lemma 3.6 tells us that $P$ is a subsemigroup of $\mathcal{T}_{X}^{a}$.

Note that if $\operatorname{rank}(f)>r$, then $f$ belongs to neither $P_{1}$ nor $P_{2}$. The next result follows from Proposition 3.2. Together with Proposition 4.1, it yields the characterisation of Green's relations on $\mathcal{T}_{X}^{a}$ given by Tsyaputa [70]; see also 25, Theorem 13.4.2].

Theorem 4.2. If $f \in \mathcal{T}_{X}^{a}$, then

(i) $R_{f}^{a}= \begin{cases}R_{f} \cap P_{1} & \text { if } f \in P_{1} \\ \{f\} & \text { if } f \in \mathcal{T}_{X} \backslash P_{1},\end{cases}$

(iii) $H_{f}^{a}= \begin{cases}H_{f} & \text { if } f \in P \\ \{f\} & \text { if } f \in \mathcal{T}_{X} \backslash P\end{cases}$

(ii) $L_{f}^{a}= \begin{cases}L_{f} \cap P_{2} & \text { if } f \in P_{2} \\ \{f\} & \text { if } f \in \mathcal{T}_{X} \backslash P_{2},\end{cases}$

(iv) $D_{f}^{a}= \begin{cases}D_{f} \cap P & \text { if } f \in P \\ L_{f}^{a} & \text { if } f \in P_{2} \backslash P_{1} \\ R_{f}^{a} & \text { if } f \in P_{1} \backslash P_{2} \\ \{f\} & \text { if } f \in \mathcal{T}_{X} \backslash\left(P_{1} \cup P_{2}\right) .\end{cases}$

The sets $P_{1}$ and $P_{2}$ are described in Proposition 4.1. In particular, $R_{f}^{a}=L_{f}^{a}=H_{f}^{a}=D_{f}^{a}=\{f\}$ if $\operatorname{rank}(f)>r$. If $f \in \mathcal{T}_{X} \backslash P$, then $H_{f}^{a}=\{f\}$ is a non-group $\mathscr{H}^{a}$-class of $\mathcal{T}_{X}^{a}$.

Remark 4.3. The article [51] characterises Green's relations and the regular elements of the more general semigroup $T(X, Y, a)$ consisting of all functions $f: X \rightarrow Y$ under the operation $f \cdot g=f \circ a \circ g$, where $a: Y \rightarrow X$ is some fixed function and $\circ$ denotes the usual composition of functions. This characterisation is, by necessity, far more complex than that given in Proposition 4.1 and Theorem 4.2 . 
Theorem 4.2 yields an intuitive picture of the Green's structure of $\mathcal{T}_{X}^{a}$. Recall that the $\mathscr{D}$-classes of $\mathcal{T}_{X}$ are precisely the sets $D_{m}=\left\{f \in \mathcal{T}_{X}: \operatorname{rank}(f)=m\right\}$ for $1 \leq m \leq n=|X|$. Each of the $\mathscr{D}$-classes $D_{r+1}, \ldots, D_{n}$ separates completely into singleton $\mathscr{D}^{a}$-classes in $\mathcal{T}_{X}^{a}$. (We will study these classes in more detail shortly.) Next, note that $D_{1} \subseteq P$ (as the constant maps clearly belong to both $P_{1}$ and $P_{2}$ ), so $D_{1}$ remains a (regular) $\mathscr{D}^{a}$-class of $\mathcal{T}_{X}^{a}$. Now fix some $2 \leq m \leq r$, and recall that we are assuming that $r<n$. The $\mathscr{D}$-class $D_{m}$ is split into a single regular $\mathscr{D}^{a}$-class, namely $D_{m} \cap P$, and a number of non-regular $\mathscr{D}^{a}$-classes. Some of these non-regular $\mathscr{D}^{a}$-classes are singletons, namely those of the form $D_{f}^{a}=\{f\}$ where $f \in D_{m}$ belongs to neither $P_{1}$ nor $P_{2}$. Some of the non-regular $\mathscr{D}^{a}$-classes consist of one non-singleton $\mathscr{L}^{a}$-class, namely those of the form $D_{f}^{a}=L_{f}^{a}=L_{f} \cap P_{2}$, where $f \in D_{m}$ belongs to $P_{2} \backslash P_{1}$; the $\mathscr{H}^{a}$-classes contained in such a $\mathscr{D}^{a}$-class are all singletons. The remaining non-regular $\mathscr{D}^{a}$-classes in $D_{m}$ consist of one non-singleton $\mathscr{R}^{a}$-class, namely those of the form $D_{f}^{a}=R_{f}^{a}=R_{f} \cap P_{1}$, where $f \in D_{m}$ belongs to $P_{1} \backslash P_{2}$; the $\mathscr{H}^{a}$-classes contained in such a $\mathscr{D}^{a}$-class are all singletons. This is all pictured (schematically) in Figure 4 , see also Figures 2 and 3.
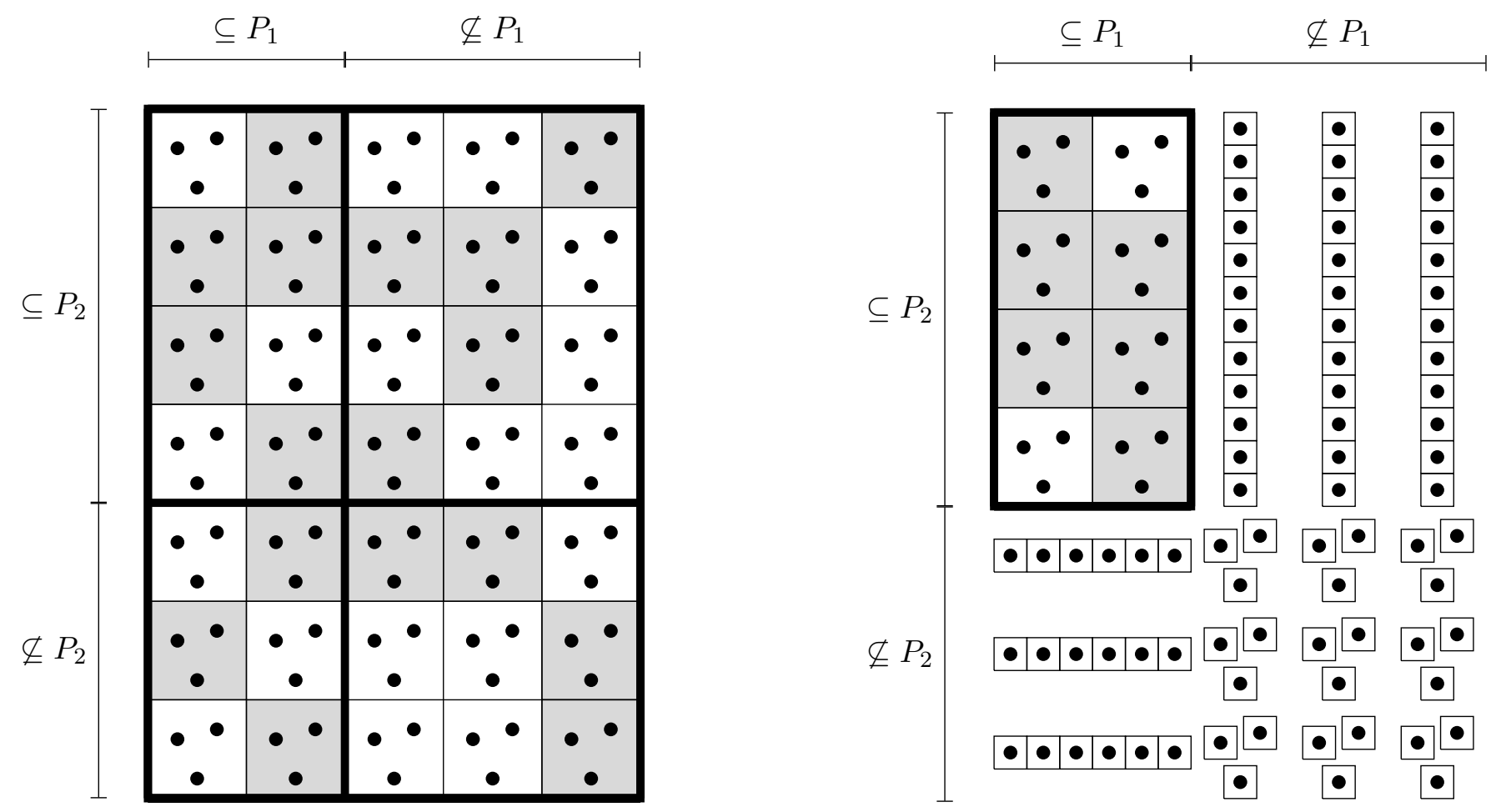

Figure 4: A schematic diagram of the way a $\mathscr{D}$-class $D_{m}$ of $\mathcal{T}_{X}$ (with $2 \leq m \leq r$ ) breaks up into $\mathscr{D}^{a}$-classes in $\mathcal{T}_{X}^{a}$. Group $\mathscr{H}$ - and $\mathscr{H}^{a}$-classes are shaded grey.

We now give some information about the order on the $\mathscr{J}^{a}=\mathscr{D}^{a}$-classes of $\mathcal{T}_{X}^{a}$. Recall that in $\mathcal{T}_{X}, D_{f} \leq D_{g}$ if and only if $\operatorname{rank}(f) \leq \operatorname{rank}(g)$. The situation is more complicated in $\mathcal{T}_{X}^{a}$.

Proposition 4.4. Let $f, g \in \mathcal{T}_{X}$. Then $D_{f}^{a} \leq D_{g}^{a}$ in $\mathcal{T}_{X}^{a}$ if and only if one of the following holds:

(i) $f=g$,

(iii) $\operatorname{im}(f) \subseteq \operatorname{im}(a g)$,

(ii) $\operatorname{rank}(f) \leq \operatorname{rank}(a g a)$,

(iv) $\operatorname{ker}(f) \supseteq \operatorname{ker}(g a)$.

The maximal $\mathscr{D}^{a}$-classes are those of the form $D_{f}^{a}=\{f\}$ where $\operatorname{rank}(f)>r$.

Proof. Note that $D_{f}^{a} \leq D_{g}^{a}$ if and only if one of the following holds:

(a) $f=g$,

(c) $f=u a g$ for some $u \in \mathcal{T}_{X}$,

(b) $f=u a g a v$ for some $u, v \in \mathcal{T}_{X}$,

(d) $f=g a v$ for some $v \in \mathcal{T}_{X}$.

We clearly have the implications (b) $\Rightarrow$ (ii), (c) $\Rightarrow$ (iii), and (d) $\Rightarrow$ (iv). Next, note that (ii) implies $D_{f} \leq D_{a g a}$ in $\mathcal{T}_{X}$, from which (b) follows. Next suppose (iii) holds. Since $\operatorname{im}(f) \subseteq \operatorname{im}(a g)$, we may write $f=\left(\begin{array}{ccc}F_{1} & \cdots & F_{m} \\ f_{1} & \cdots & f_{m}\end{array}\right)$ and $a g=\left(\begin{array}{cccccc}G_{1} & \cdots & G_{m} & G_{m+1} & \cdots & G_{l} \\ f_{1} & \cdots & f_{m} & g_{m+1} & \cdots & g_{l}\end{array}\right)$. For $i \in \mathbf{m}$, let $g_{i} \in G_{i}$. We then have $f=u a g$, where 
$u=\left(\begin{array}{ccc}F_{1} & \cdots & F_{m} \\ g_{1} & \cdots & g_{m}\end{array}\right)$, giving (c). Finally, suppose (iv) holds, and write $f=\left(\begin{array}{ccc}F_{1} & \cdots & F_{m} \\ f_{1} & \cdots & f_{m}\end{array}\right)$ and $g a=\left(\begin{array}{lll}G_{1} & \cdots & G_{l} \\ g_{1} & \cdots & g_{l}\end{array}\right)$. Since $\operatorname{ker}(f) \supseteq \operatorname{ker}(g a)$, there is a surjective function $q: \mathbf{l} \rightarrow \mathbf{m}$ such that $G_{i} \subseteq F_{i q}$ for all $i$. We see then that $f=$ gav, where $v \in \mathcal{T}_{X}$ is any transformation that extends the partial map $\left(\begin{array}{ccc}g_{1} & \cdots & g_{l} \\ f_{1 q} & \cdots & f_{l_{q}}\end{array}\right)$, giving (d).

To prove the statement concerning maximal $\mathscr{D}^{a}$-classes, let $f \in \mathcal{T}_{X}$. If $\operatorname{rank}(f) \leq r$, then $\operatorname{rank}(f) \leq$ $\operatorname{rank}(a)=\operatorname{rank}(a 1 a)$, so that $D_{f}^{a}<D_{1}^{a}=\{1\}$, whence $D_{f}^{a}$ is not maximal. (Here, $1 \in \mathcal{T}_{X}$ denotes the identity element of $\mathcal{T}_{X}$, namely the identity map $X \rightarrow X$.) On the other hand, suppose $\operatorname{rank}(f)>r$ and that $D_{f}^{a} \leq D_{g}^{a}$. Then (ii) does not hold, $\operatorname{since} \operatorname{rank}(a g a) \leq \operatorname{rank}(a)=r<\operatorname{rank}(f)$. Similarly, $\operatorname{rank}(a g)<\operatorname{rank}(f)$ and $\operatorname{rank}(g a)<\operatorname{rank}(f)$, so neither (iii) nor (iv) holds. Having eliminated (ii-iv), we deduce that (i) must hold; that is, $f=g$, so $D_{f}^{a}=\{f\}$ is maximal.

Remark 4.5. If $r=\operatorname{rank}(a)=1$, then $\mathcal{T}_{X}^{a}$ has a very simple structure, as may be deduced from Theorem 4.2 and Proposition 4.4; see Figure 5 for an illustration in the case $n=|X|=3$. This structure may also be observed directly. For $x \in X$, denote by $c_{x} \in \mathcal{T}_{X}$ the constant map with image $\{x\}$. If $a=c_{x}$, then for all $f, g \in \mathcal{T}_{X}, f \star g=f c_{x} g=c_{x} g=c_{x g}$.

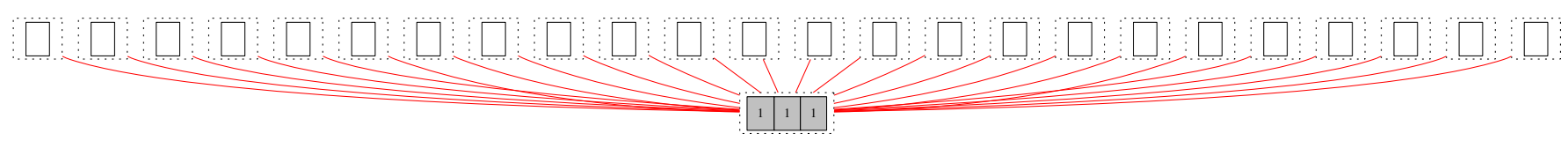

Figure 5: Egg box diagram of the variant semigroup $\mathcal{T}_{3}^{a}$, where $a=[1,1,1]$.

The description of the maximal $\mathscr{D}^{a}$-classes from Proposition 4.4 allows us to obtain information about $\operatorname{rank}\left(\mathcal{T}_{X}^{a}\right)$. In order to avoid confusion when discussing generation, if $U \subseteq \mathcal{T}_{X}$, we will write $\langle U\rangle$ (resp., $\langle U\rangle_{a}$ ) for the subsemigroup of $\mathcal{T}_{X}$ (resp., $\mathcal{T}_{X}^{a}$ ) generated by $U$, which consists of all products $u_{1} \cdots u_{k}$ (resp., $\left.u_{1} \star \cdots \star u_{k}\right)$, where $k \geq 1$ and $u_{1}, \ldots, u_{k} \in U$.

Theorem 4.6. Let $M=\left\{f \in \mathcal{T}_{X}: \operatorname{rank}(f)>r\right\}$. Then $\mathcal{T}_{X}^{a}=\langle M\rangle_{a}$. Further, any generating set for $\mathcal{T}_{X}^{a}$ contains $M$. Consequently, $M$ is the unique minimal (with respect to containment or size) generating set of $\mathcal{T}_{X}^{a}$, and

$$
\operatorname{rank}\left(\mathcal{T}_{X}^{a}\right)=|M|=\sum_{m=r+1}^{n} S(n, m)\left(\begin{array}{c}
n \\
m
\end{array}\right) m !,
$$

where $S(n, m)$ denotes the (unsigned) Stirling number of the second kind.

Proof. Consider the statement:

$$
H(m): \quad\langle M\rangle_{a} \text { contains } D_{m} \cup \cdots \cup D_{n}=\left\{f \in \mathcal{T}_{X}: \operatorname{rank}(f) \geq m\right\} .
$$

Since $H(1)$ says that $\mathcal{T}_{X}^{a}=\langle M\rangle_{a}$, it suffices to show that $H(m)$ is true for all $m \in \mathbf{n}$. We do this by (reverse) induction on $m$. Note that $M=D_{r+1} \cup \cdots \cup D_{n}$, so $H(m)$ is clearly true for $m \geq r+1$. Now suppose $H(m+1)$ is true for some $1 \leq m \leq r$. Let $f \in D_{m}$, and write $f=\left(\begin{array}{lll}F_{1} & \cdots & F_{m} \\ f_{1} & \cdots & f_{m}\end{array}\right)$. Since $m \leq r<n$, we may assume that $\left|F_{1}\right| \geq 2$. Choose some non-trivial partition $F_{1}=F_{1}^{\prime} \sqcup F_{1}^{\prime \prime}$. Without loss of generality, we may also assume that $\left|A_{1}\right| \geq 2$. Choose some $a_{1}^{\prime} \in A_{1} \backslash\left\{a_{1}\right\}$, and put $g=\left(\begin{array}{ccccc}F_{1}^{\prime} & F_{1}^{\prime \prime} & F_{2} & \cdots & F_{m} \\ a_{1}^{\prime} & a_{1} & a_{2} & \cdots & a_{m}\end{array}\right)$. So $g \in\langle M\rangle_{a}$, by the induction hypothesis. Also, let $h \in \mathcal{S}_{X} \subseteq M$ be any permutation that extends the partial map $\left(\begin{array}{llll}a_{1} & \cdots & a_{m} \\ f_{1} & \cdots & f_{m}\end{array}\right)$. Then $f=g a h=g \star h \in\langle M\rangle_{a}$, so $H(m)$ is true, completing the inductive step.

Any $f \in M$ belongs to a non-group, maximal $\mathscr{D}^{a}$-class, so it follows that any generating set of $\mathcal{T}_{X}^{a}$ must contain $M$. This tells us that $M$ is the minimal generating set with respect to both size and containment, and that $\operatorname{rank}\left(\mathcal{T}_{X}^{a}\right)=|M|$. The formula for $|M|$ follows from the well-known fact that $\left|D_{m}\right|=S(n, m)\left(\begin{array}{c}n \\ m\end{array}\right) m$ ! for any $m \in \mathbf{n}[25]$. This completes the proof.

Remark 4.7. It seems noteworthy that $\operatorname{rank}\left(\mathcal{T}_{X}^{a}\right)$ depends only on $r=\operatorname{rank}(a)$, and not on the sizes $\lambda_{1}, \ldots, \lambda_{r}$ of the kernel-classes of $a$. See also Theorems 5.18, 6.8 and 7.4 . 
The description of the order on $\mathscr{D}^{a}$-classes of $\mathcal{T}_{X}^{a}$ from Proposition 4.4 may be simplified in the case that one of $f, g$ is regular.

Proposition 4.8. Let $f, g \in \mathcal{T}_{X}$.

(i) If $f \in P$, then $D_{f}^{a} \leq D_{g}^{a}$ if and only if $\operatorname{rank}(f) \leq \operatorname{rank}(a g a)$.

(ii) If $g \in P$, then $D_{f}^{a} \leq D_{g}^{a}$ if and only if $\operatorname{rank}(f) \leq \operatorname{rank}(g)$.

The regular $\mathscr{D}^{a}$-classes of $\mathcal{T}_{X}^{a}$ form a chain: $D_{1}^{a}<\cdots<D_{r}^{a}$, where $D_{m}^{a}=\{f \in P: \operatorname{rank}(f)=m\}$ for $m \in \mathbf{r}$.

Proof. As in the proof of Proposition 4.4, $D_{f}^{a} \leq D_{g}^{a}$ if and only if one of the following holds:

(a) $f=g$,

(b) $f=u a g a v$ for some $u, v \in \mathcal{T}_{X}$, (c) $f=u a g$ for some $u \in \mathcal{T}_{X}$,

(d) $f=g a v$ for some $v \in \mathcal{T}_{X}$.

Suppose first that $f \in P$, so $f=$ fahaf for some $h \in \mathcal{T}_{X}$. Then (a) implies $f=$ fah(aga)haf, (c) implies $f=u($ aga $h a f$, and (d) implies $f=f a h(a g a) v$. So, in each of cases (a-d), we deduce that $\operatorname{rank}(f) \leq \operatorname{rank}(a g a)$. We have already observed that $\operatorname{rank}(f) \leq \operatorname{rank}(a g a)$ implies $D_{f}^{a} \leq D_{g}^{a}$.

Next, suppose $g \in P$. Since $\operatorname{rank}(a g)=\operatorname{rank}(g a)=\operatorname{rank}(a g a)=\operatorname{rank}(g)$, each of $(\mathrm{a}-\mathrm{d}) \operatorname{implies} \operatorname{rank}(f) \leq$ $\operatorname{rank}(g)$. If $\operatorname{rank}(f) \leq \operatorname{rank}(g)=\operatorname{rank}(a g a)$, then we already know that $D_{f}^{a} \leq D_{g}^{a}$. The statement about regular $\mathscr{D}^{a}$-classes follows quickly from (ii).

Proposition 4.8 gives us some more partial information about the location of the "fragmented" $\mathscr{D}^{a}$-classes (see Figure 4). Specifically, a non-regular $\mathscr{D}^{a}$-class $D_{f}^{a}$ with $\operatorname{rank}(f)=m \leq r$ sits below $D_{m}^{a}$. However, $D_{f}^{a}$ may or may not sit above $D_{m-1}^{a}$; this depends on $\operatorname{rank}(a f a)$. For example, if $a=[1,1,1,4,5]$ and $f=[1,2,3,1,1]$, then $D_{f}^{a}$ sits between $D_{1}^{a}$ and $D_{3}^{a}$ but not above $D_{2}^{a}$ in $\mathcal{T}_{5}^{a}$. While it is extremely difficult to enumerate all $\mathscr{D}^{a}$-classes (even maximal ones) that sit above $D_{m}^{a}$ but not $D_{m+1}^{a}$, where $m \in \mathbf{r}$ is arbitrary, we can enumerate those that sit right at the top of the picture, above the highest regular $\mathscr{D}^{a}$-class, $D_{r}^{a}$. Recall that $\Lambda=\lambda_{1} \cdots \lambda_{r}$, where $\lambda_{i}=\left|A_{i}\right|$.

Proposition 4.9. A maximal $\mathscr{D}^{a}$-class $D_{f}^{a}=\{f\}$ sits above $D_{r}^{a}$ in the ordering of $\mathscr{D}^{a}$-classes in $\mathcal{T}_{X}^{a}$ if and only if $\operatorname{rank}(a f a)=r<\operatorname{rank}(f)$. The number of such $\mathscr{D}^{a}$-classes is equal to $\left(n^{n-r}-r^{n-r}\right) r ! \Lambda$.

Proof. The first statement follows from Proposition 4.8(i). It remains to count the number of transformations $f \in \mathcal{T}_{X}$ satisfying $\operatorname{rank}(a f a)=r<\operatorname{rank}(f)$. Note that such an $f$ maps $A$ to a cross-section of $\alpha=\operatorname{ker}(a)$. The number of cross-sections of $\alpha$ is $\lambda_{1} \cdots \lambda_{r}=\Lambda$, and once such a cross-section $B=\left\{b_{1}, \ldots, b_{r}\right\}$ is chosen, there are $r$ ! ways to choose $\left.f\right|_{A}$ (which maps $A$ bijectively to $B$ ). There are $n^{n-r}-r^{n-r}$ ways to extend $\left.f\right|_{A}$ to $f \in \mathcal{T}_{X}$ with $\operatorname{rank}(f)>r$.

\section{The regular semigroup $\operatorname{Reg}\left(\mathcal{T}_{X}^{a}\right)$}

In this section, we study the subsemigroup

$$
P=\operatorname{Reg}\left(\mathcal{T}_{X}^{a}\right)=\left\{f \in \mathcal{T}_{X}: \operatorname{rank}(a f a)=\operatorname{rank}(f)\right\},
$$

consisting of all regular elements of $\mathcal{T}_{X}^{a}$. Key results include a description of $P$ as a subdirect product of the well-known semigroups $\operatorname{Reg}(\mathcal{T}(X, A))$ and $\operatorname{Reg}(\mathcal{T}(X, \alpha))$ (see below for definitions), a realisation of $P$ as a kind of "inflation" of $\mathcal{T}_{A}$, combinatorial results on the number of Green's classes of certain types, and calculations of $|P|$ and $\operatorname{rank}(P)$. As before, we assume that

$$
a=\left(\begin{array}{lll}
A_{1} & \cdots & A_{r} \\
a_{1} & \cdots & a_{r}
\end{array}\right)
$$


is an idempotent with $\operatorname{rank}(a)=r<n$, and we continue to write $A=\operatorname{im}(a), \alpha=\operatorname{ker}(a), \lambda_{i}=\left|A_{i}\right|$, and so on. By Theorem 4.2, we see that $\operatorname{Reg}\left(\mathcal{T}_{X}^{a}\right)=D_{1}$ is a right zero semigroup in the case $r=1$ (see also Remark 4.5 and Figure 5), in which case, all the problems we consider become trivial. So for the duration of this section, we will assume that $1<r<n$.

Figures 2 and 3 picture the variant $\mathcal{T}_{4}^{a}$ with respect to various transformations $a \in \mathcal{T}_{4}$, and one may see the regular subsemigroup $\operatorname{Reg}\left(\mathcal{T}_{4}^{a}\right)$ in each case as the collection of $\mathscr{D}^{a}$-classes containing groups (shaded cells). Figure 6 pictures $\operatorname{Reg}\left(\mathcal{T}_{5}^{a}\right)$ for various choices of $a \in \mathcal{T}_{5}$ with $\operatorname{rank}(a) \leq 4$. When one compares Figure 6 with Figure 1, which pictures the semigroups $\mathcal{T}_{1}, \mathcal{T}_{2}, \mathcal{T}_{3}, \mathcal{T}_{4}$, a striking pattern seems to emerge. In each case, $P=\operatorname{Reg}\left(\mathcal{T}_{5}^{a}\right)$ looks like some kind of "inflation" of $\mathcal{T}_{r}$ (where $r=\operatorname{rank}(a)$ ), in the sense that one may begin with an egg box diagram of $\mathcal{T}_{r}$ and then subdivide the cells in some way to obtain an egg box diagram of $P$; further, it appears that the subdivision is done in such a way that group (resp., non-group) $\mathscr{H}$-classes of $\mathcal{T}_{m}$ become rectangular arrays of group (resp., non-group) $\mathscr{H}^{a}$-classes of $P$, although the reason for the exact number of subdivisions applied to each cell may not yet be apparent. One of the goals of this section is to explain the reason for this phenomenon.

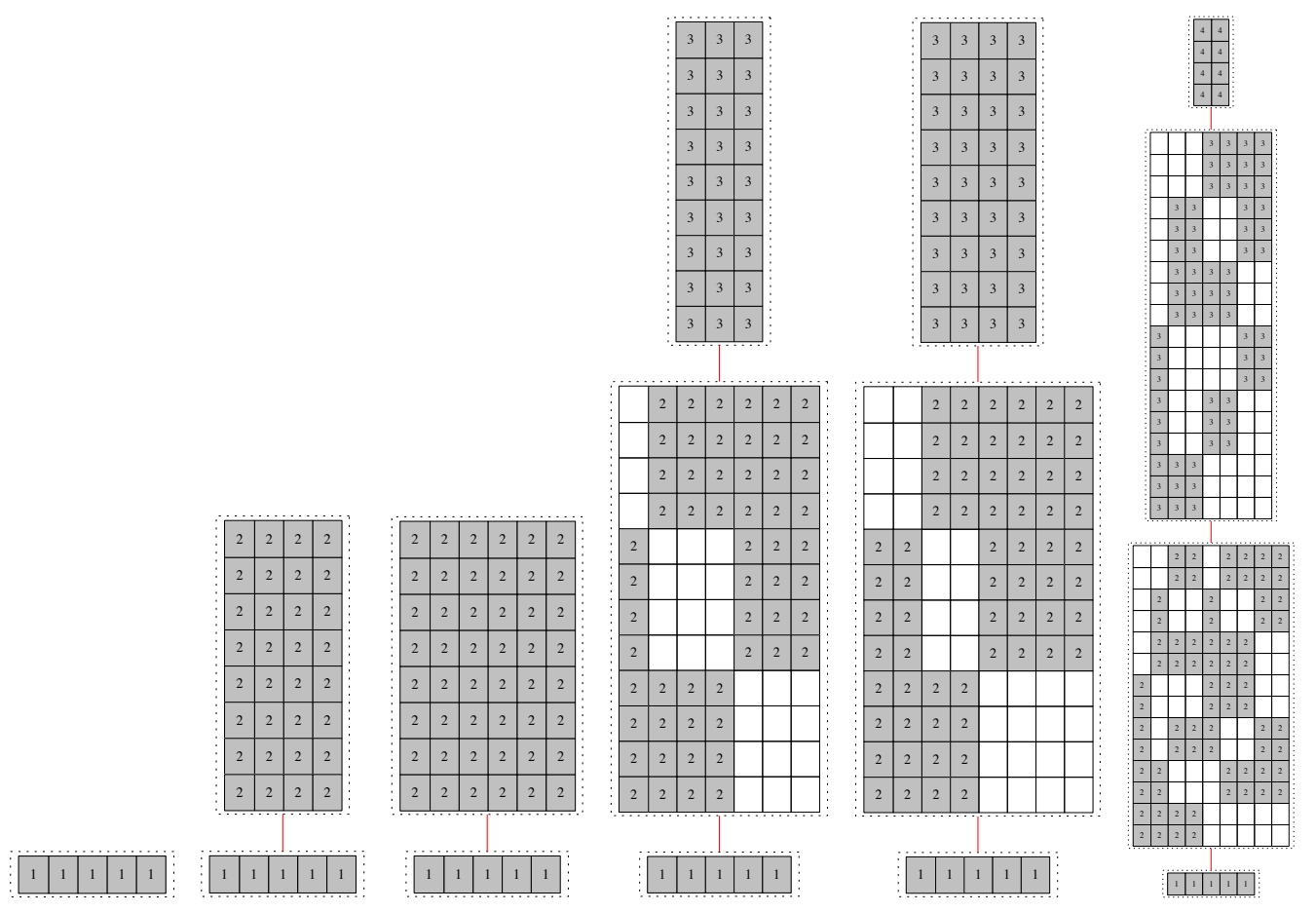

Figure 6: Egg box diagrams of the regular subsemigroups $P=\operatorname{Reg}\left(\mathcal{T}_{5}^{a}\right)$ in the cases (from left to right): $a=[1,1,1,1,1], a=[1,2,2,2,2], a=[1,1,2,2,2], a=[1,2,3,3,3], a=[1,2,2,3,3], a=[1,2,3,4,4]$.

Now, Theorem 4.2 enables us to immediately describe Green's relations on $P=\operatorname{Reg}\left(\mathcal{T}_{X}^{a}\right)$. Since $P$ is a regular subsemigroup of $\mathcal{T}_{X}^{a}$, the $\mathscr{R}, \mathscr{L}, \mathscr{H}$ relations on $P$ are just the restrictions of the corresponding relations on $\mathcal{T}_{X}^{a}$ (see for example [37, 42]), and it is easy to check that this is also true for the $\mathscr{D}=\mathscr{J}$ relation in this case. So if $\mathscr{K}$ is one of $\mathscr{R}, \mathscr{L}, \mathscr{H}, \mathscr{D}$, we will continue to write $\mathscr{K}^{a}$ for the $\mathscr{K}$ relation on $P$, and write $K_{f}^{a}$ for the $\mathscr{K}^{a}$-class of $f$ in $P$ for any $f \in P$.

Corollary 5.1. If $f \in P$, then

(i) $R_{f}^{a}=R_{f} \cap P=\{g \in P: \operatorname{ker}(f)=\operatorname{ker}(g)\}$,

(ii) $L_{f}^{a}=L_{f} \cap P=\{g \in P: \operatorname{im}(f)=\operatorname{im}(g)\}$,

(iii) $H_{f}^{a}=H_{f} \cap P=\{g \in P: \operatorname{ker}(f)=\operatorname{ker}(g)$ and $\operatorname{im}(f)=\operatorname{im}(g)\}$,

(iv) $D_{f}^{a}=D_{f} \cap P=\{g \in P: \operatorname{rank}(f)=\operatorname{rank}(g)\}$.

The $\mathscr{D}^{a}$-classes of $P$ form a chain: $D_{1}^{a}<\cdots<D_{r}^{a}$, where $D_{m}^{a}=\{f \in P: \operatorname{rank}(f)=m\}$ for each $m \in \mathbf{r}$. 
Corollary 5.1 gives a descriptive characterisation of Green's relations on $P=\operatorname{Reg}\left(\mathcal{T}_{X}^{a}\right)$; in particular, it relates each relation $\mathscr{K}^{a}$ on $P$ directly to the relation $\mathscr{K}$ on $\mathcal{T}_{X}$. But it says nothing about why $P$ appears to be an inflated version of $\mathcal{T}_{r}$. In order to explain this phenomenon, we must further explore the structure of $P$. We will do this by examining a certain relationship between $P$ and $\mathcal{T}_{A}$, the full transformation semigroup on $A=\operatorname{im}(a)$, as well as some other well-known subsemigroups of $\mathcal{T}_{X}$. Recall that the sets

$$
\mathcal{T}(X, A)=\left\{f \in \mathcal{T}_{X}: \operatorname{im}(f) \subseteq A\right\} \quad \text { and } \quad \mathcal{T}(X, \alpha)=\left\{f \in \mathcal{T}_{X}: \operatorname{ker}(f) \supseteq \alpha\right\}
$$

are subsemigroups of $\mathcal{T}_{X}$. These semigroups have been studied extensively in the literature, where they are typically referred to as semigroups of transformations of restricted range or restricted kernel (respectively); see for example [56,62,64,66], and references therein.

Remark 5.2. Note that $\mathcal{T}(X, A)=\mathcal{T}_{X} a$ and $\mathcal{T}(X, \alpha)=a \mathcal{T}_{X}$, as subsemigroups of $\mathcal{T}_{X}$ (with respect to the usual operation). Indeed, the maps

$$
\rho_{a}: \mathcal{T}_{X}^{a} \rightarrow \mathcal{T}(X, A)=\mathcal{T}_{X} a: f \mapsto f a \quad \text { and } \quad \lambda_{a}: \mathcal{T}_{X}^{a} \rightarrow \mathcal{T}(X, \alpha)=a \mathcal{T}_{X}: f \mapsto a f
$$

are easily seen to be epimorphisms. Since products in $\mathcal{T}(X, A)=\mathcal{T}_{X} a$ and $\mathcal{T}(X, \alpha)=a \mathcal{T}_{X}$ are found by forming expressions such as faga and afag (respectively), it should be no surprise that these semigroups play a role in an investigation of the structure of $\mathcal{T}_{X}^{a}$. Since we are assuming $a$ is an idempotent, it also follows that $\mathcal{T}(X, A)=\mathcal{T}_{X} \star a$ and $\mathcal{T}(X, \alpha)=a \star \mathcal{T}_{X}$, as subsemigroups of $\mathcal{T}_{X}^{a}$ (with respect to the $\star$ operation). As noted in [25], if $S$ is either $\mathcal{T}(X, A)$ or $\mathcal{T}(X, \alpha)$, the semigroups $S^{a}$ and $S$ are precisely the same object; that is, $f \star g=f g$ for all $f, g \in S$. (This is because $a$, being an idempotent of $\mathcal{T}_{X}$, is a mididentity of both $a \mathcal{T}_{X}$ and $\left.\mathcal{T}_{X} a.\right)$

The regular elements of the semigroups $\mathcal{T}(X, A)$ and $\mathcal{T}(X, \alpha)$ have been described in [63] and [56], respectively; in terms of our notation, the description is as follows. Recall that

$$
P_{1}=\left\{f \in \mathcal{T}_{X}: \alpha \text { separates } \operatorname{im}(f)\right\} \quad \text { and } \quad P_{2}=\left\{f \in \mathcal{T}_{X}: A \text { saturates } \operatorname{ker}(f)\right\} .
$$

Proposition 5.3 (Sanwong and Sommanee $[63]$; Mendes-Gonçalves and Sullivan [56]). The regular elements of $\mathcal{T}(X, A)$ and $\mathcal{T}(X, \alpha)$ are precisely the sets

$$
\operatorname{Reg}(\mathcal{T}(X, A))=\mathcal{T}(X, A) \cap P_{2} \quad \text { and } \quad \operatorname{Reg}(\mathcal{T}(X, \alpha))=\mathcal{T}(X, \alpha) \cap P_{1} .
$$

The next two propositions are the main structural results of this section.

Proposition 5.4. There is a well-defined monomorphism

$$
\psi: \operatorname{Reg}\left(\mathcal{T}_{X}^{a}\right) \rightarrow \operatorname{Reg}(\mathcal{T}(X, A)) \times \operatorname{Reg}(\mathcal{T}(X, \alpha)): f \mapsto(f a, a f) .
$$

The image of $\psi$ is the set

$$
\operatorname{im}(\psi)=\left\{(g, h) \in \operatorname{Reg}(\mathcal{T}(X, A)) \times \operatorname{Reg}(\mathcal{T}(X, \alpha)): \operatorname{rank}(g)=\operatorname{rank}(h),\left.g\right|_{A}=\left.(h a)\right|_{A}\right\} .
$$

In particular, $\operatorname{Reg}\left(\mathcal{T}_{X}^{a}\right)$ is (isomorphic to) a subdirect product of $\operatorname{Reg}(\mathcal{T}(X, A))$ and $\operatorname{Reg}(\mathcal{T}(X, \alpha))$.

Proof. Let $f \in P=\operatorname{Reg}\left(\mathcal{T}_{X}^{a}\right)$. Since $P$ is a subsemigroup of $\mathcal{T}_{X}^{a}$, we have $f a=f a a=f \star a \in P$; in particular, $f a \in \mathcal{T}_{X} a \cap P_{2}=\operatorname{Reg}(\mathcal{T}(X, A))$. A similar calculation shows that af $\in \operatorname{Reg}(\mathcal{T}(X, \alpha))$. If $f, g \in P$, then $(f \star g) \psi=(f a g) \psi=((f a g) a, a(f a g))=(f a, a f)(g a, a g)=(f \psi)(g \psi)$, so $\psi$ is a homomorphism. Suppose now that $f, g \in P$ are such that $f \psi=g \psi$. So $f a=g a$ and $a f=a g$, and we must show that $f=g$. Since $A$ saturates $\operatorname{ker}(f)$ and $\operatorname{ker}(g)$, it suffices to show that $\operatorname{ker}(f)=\operatorname{ker}(g)$ and $\left.f\right|_{A}=\left.g\right|_{A}$. Now, for any $x \in A$, we have $x f=x a f=x a g=x g$, so $\left.f\right|_{A}=\left.g\right|_{A}$. Also note that since $f \in P_{1}$, $\operatorname{ker}(f a)=\operatorname{ker}(f)$. Similarly, $\operatorname{ker}(g a)=\operatorname{ker}(g)$. Since $f a=g a$, it follows that $\operatorname{ker}(f)=\operatorname{ker}(g)$. As noted above, this completes the proof that $\psi$ is injective.

To prove the statement concerning $\operatorname{im}(\psi)$, first suppose $f \in P$ and put $g=f a$ and $h=a f$. Since $f \in P=P_{1} \cap P_{2}$, Proposition 4.1 gives $\operatorname{rank}(g)=\operatorname{rank}(f)=\operatorname{rank}(h)$. Since $a$ maps $A$ identically, it follows 
that $\left.(a q)\right|_{A}=\left.q\right|_{A}$ for all $q \in \mathcal{T}_{X}$. In particular, $\left.(h a)\right|_{A}=\left.(a f a)\right|_{A}=\left.(f a)\right|_{A}=\left.g\right|_{A}$. Conversely, suppose $g \in \operatorname{Reg}(\mathcal{T}(X, A))$ and $h \in \operatorname{Reg}(\mathcal{T}(X, \alpha))$ satisfy $\operatorname{rank}(g)=\operatorname{rank}(h)$ and $\left.g\right|_{A}=\left.(h a)\right|_{A}$. Put $m=\operatorname{rank}(g)$, and write $g=\left(\begin{array}{ccc}G_{1} & \ldots & G_{m} \\ a_{k_{1}} & \cdots & a_{k_{m}}\end{array}\right)$ and $h=\left(\begin{array}{ccc}H_{1} & \cdots & H_{m} \\ h_{1} & \cdots & h_{m}\end{array}\right)$, noting that $\operatorname{im}(g) \subseteq A$. Also, since $h \in \mathcal{T}(X, \alpha)$, there is a partition $\mathbf{r}=I_{1} \sqcup \cdots \sqcup I_{m}$ such that $H_{j}=\bigcup_{i \in I_{j}} A_{i}$ for each $j$. Now, since $g \in \operatorname{Reg}(\mathcal{T}(X, A))$, $A$ saturates $\operatorname{ker}(g)$, so it follows that $G_{i} \cap A \neq \emptyset$ for all $i$. Thus, $\left.g\right|_{A}=\left(\begin{array}{ccc}G_{1} \cap A & \ldots & G_{m} \cap A \\ a_{k_{1}} & \ldots & a_{k_{m}}\end{array}\right)$. For each $i \in \mathbf{m}$, let $l_{i} \in \mathbf{r}$ be such that $h_{i} \in A_{l_{i}}$. Since $h \in \operatorname{Reg}(\mathcal{T}(X, \alpha))$, $\alpha$ separates $\operatorname{im}(h)$, so $l_{1}, \ldots, l_{m}$ are distinct. It follows that $h a=\left(\begin{array}{ccc}H_{1} & \cdots & H_{m} \\ a_{l_{1}} & \cdots & a_{l_{m}}\end{array}\right)$. Since each $H_{i} \cap A$ is non-empty (as $H_{i}$ is a union of $\alpha$-classes, each of which contains an element of $A$ ), we have $\left.(h a)\right|_{A}=\left(\begin{array}{cccc}H_{1} \cap A & \ldots & H_{m} \cap A \\ a_{l_{1}} & \ldots & a_{l_{m}}\end{array}\right)$. But $\left.g\right|_{A}=\left.(h a)\right|_{A}$, so (reordering if necessary), it follows that $l_{i}=k_{i}$ and $H_{i} \cap A=G_{i} \cap A$ for each $i$. In particular, $h_{i} \in A_{l_{i}}=A_{k_{i}}$ for each $i$. Now put $f=\left(\begin{array}{ccc}G_{1} & \cdots & G_{m} \\ h_{1} & \cdots & h_{m}\end{array}\right)$. Since $\operatorname{ker}(f)=\operatorname{ker}(g)$ and $\operatorname{im}(f)=\operatorname{im}(h)$, we see that $f \in P$. It is clear that $f a=g$. We also have $a f=h$ since, for all $j$,

$$
H_{j} a f=\left(\bigcup_{i \in I_{j}} A_{i}\right) a f=\left\{a_{i}: i \in I_{j}\right\} f=\left(H_{j} \cap A\right) f=\left(G_{j} \cap A\right) f=h_{j} .
$$

It follows that $(g, h)=f \psi$. Finally, suppose $g \in \operatorname{Reg}(\mathcal{T}(X, A))$ and $h \in \operatorname{Reg}(\mathcal{T}(X, \alpha))$. To prove the statement about $\operatorname{Reg}\left(\mathcal{T}_{X}^{a}\right)$ being a subdirect product, we must show that there exist $h^{\prime} \in \operatorname{Reg}(\mathcal{T}(X, \alpha))$ and $g^{\prime} \in \operatorname{Reg}(\mathcal{T}(X, A))$ such that $\left(g, h^{\prime}\right),\left(g^{\prime}, h\right) \in \operatorname{im}(\psi)$. First note that $g \in P_{2}$ by Proposition 5.3. But also $\mathcal{T}(X, A) \subseteq P_{1}$, so $g \in P$, and $(g, a g)=(g a, a g)=g \psi$, so we may take $h^{\prime}=a g$. Similarly, $h \in P$ and $(h a, h)=h \psi$, and we take $g^{\prime}=h a$. This completes the proof.

The homomorphism $\psi$ from the previous result is built up out of the two homomorphisms

$$
\psi_{1}: P \rightarrow \operatorname{Reg}(\mathcal{T}(X, A)): f \mapsto f a \quad \text { and } \quad \psi_{2}: P \rightarrow \operatorname{Reg}(\mathcal{T}(X, \alpha)): f \mapsto a f,
$$

which are the restrictions to $P=\operatorname{Reg}\left(\mathcal{T}_{X}^{a}\right)$ of the epimorphisms $\lambda_{a}$ and $\rho_{a}$ from Remark 5.2. The last paragraph of the previous proof shows that $\psi_{1}$ and $\psi_{2}$ are epimorphisms, indeed projections, since $P$ contains both $\operatorname{Reg}(\mathcal{T}(X, A))$ and $\operatorname{Reg}(\mathcal{T}(X, \alpha))$, and $\psi_{1}$ (resp., $\left.\psi_{2}\right)$ maps $\operatorname{Reg}(\mathcal{T}(X, A))$ (resp., $\left.\operatorname{Reg}(\mathcal{T}(X, \alpha))\right)$ identically.

Proposition 5.5. The maps

$$
\phi_{1}: \operatorname{Reg}(\mathcal{T}(X, A)) \rightarrow \mathcal{T}_{A}:\left.g \mapsto g\right|_{A} \quad \text { and } \quad \phi_{2}: \operatorname{Reg}(\mathcal{T}(X, \alpha)) \rightarrow \mathcal{T}_{A}:\left.g \mapsto(g a)\right|_{A}
$$

are epimorphisms, and the following diagram commutes:

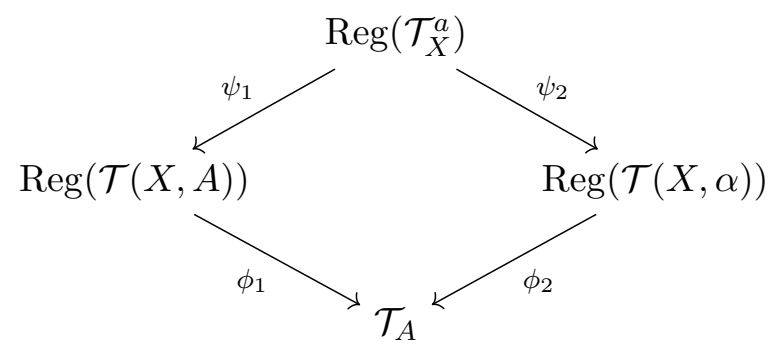

Further, the induced map $\operatorname{Reg}\left(\mathcal{T}_{X}^{a}\right) \rightarrow \mathcal{T}_{A}$ is an epimorphism.

Proof. Clearly $\phi_{1}$ and $\phi_{2}$ map their domains into $\mathcal{T}_{A}$. Note that for any $f \in \mathcal{T}_{X},\left.f\right|_{A}=\mathrm{id}{ }_{A} \circ f$, where $\operatorname{id}_{A}$ is the restriction of the identity map to $A$, and o denotes the usual composition of partial functions. So, if $g, h \in \operatorname{Reg}(\mathcal{T}(X, A))$, then

$$
(g h) \phi_{1}=\operatorname{id}_{A} \circ g \circ h=\operatorname{id}_{A} \circ g \circ \operatorname{id}_{A} \circ h=\left(g \phi_{1}\right)\left(h \phi_{1}\right),
$$

since $g=g \circ \operatorname{id}_{A}$ as $\operatorname{im}(g) \subseteq A$. If $g, h \in \operatorname{Reg}(\mathcal{T}(X, \alpha))$, then

$$
(g h) \phi_{2}=\operatorname{id}_{A} \circ g \circ h \circ a=\operatorname{id}_{A} \circ g \circ a \circ h \circ a=\operatorname{id}_{A} \circ g \circ a \circ \operatorname{id}_{A} \circ h \circ a=\left(g \phi_{2}\right)\left(h \phi_{2}\right),
$$


since $h=a \circ h$ as $\alpha \subseteq \operatorname{ker}(h)$, and $a=a \circ \mathrm{oid}_{A}$. So $\phi_{1}$ and $\phi_{2}$ are homomorphisms. That the diagram commutes follows from the fact that $\left.(a f a)\right|_{A}=\left.(f a)\right|_{A}$ for all $f \in P$, as observed in the proof of Proposition 5.4. Finally, let $q \in \mathcal{T}_{A}$, and write $q=\left(\begin{array}{ccc}a_{1} & \cdots & a_{r} \\ a_{k_{1}} & a_{k_{r}}\end{array}\right)$. (This notation is not supposed to imply that $k_{1}, \ldots, k_{r}$ are distinct.) Put $f=\left(\begin{array}{ccc}A_{1} & \ldots & A_{r} \\ a_{k_{1}} & \cdots & a_{k_{r}}\end{array}\right)$. Then clearly, $f \in \operatorname{Reg}(\mathcal{T}(X, A)) \cap \operatorname{Reg}(\mathcal{T}(X, \alpha))$ and $q=f \phi_{1}=f \phi_{2}$, showing that $\phi_{1}$ and $\phi_{2}$ are surjective. Note that, in fact, $f \in P$ and $q=f \phi_{1}=(f a) \phi_{1}=f\left(\psi_{1} \phi_{1}\right)$, showing that $\psi_{1} \phi_{1}$ is surjective, and completing the proof.

Remark 5.6. The previous result displays the structure of $P=\operatorname{Reg}\left(\mathcal{T}_{X}^{a}\right) \cong \operatorname{im}(\psi)$ as a pullback product of $\operatorname{Reg}(\mathcal{T}(X, A))$ and $\operatorname{Reg}(\mathcal{T}(X, \alpha))$ with respect to $\mathcal{T}_{A}$. Namely, $\operatorname{im}(\psi)$ consists of all pairs $(g, h)$ such that $g \phi_{1}=h \phi_{2}$. Pullback products have been studied in various contexts in universal algebra and semigroup theory (where they are sometimes referred to as spined products); see for example [12, 13, 20, 24, 48.

From now on, we will denote by $\phi=\psi_{1} \phi_{1}=\psi_{2} \phi_{2}$ the epimorphism $P \rightarrow \mathcal{T}_{A}:\left.f \mapsto(f a)\right|_{A}$. If $f \in P$, we will write $\bar{f}=f \phi \in \mathcal{T}_{A}$. If $U \subseteq P$, we write $\bar{U}=\{\bar{u}: u \in U\} \subseteq \mathcal{T}_{A}$.

We now show how $\phi: P \rightarrow \mathcal{T}_{A}$ may be used to relate Greens relations on the semigroups $P$ and $\mathcal{T}_{A}$. If $f, g \in P$ and $\mathscr{K}$ is one of $\mathscr{L}, \mathscr{R}, \mathscr{H}, \mathscr{D}$, we say $f \widehat{\mathscr{K}} g$ if $\bar{f} \mathscr{K} \bar{g}$ in $\mathcal{T}_{A}$. Denote by $\widehat{K}_{f}=K_{\bar{f}} \phi^{-1}$ the $\widehat{\mathscr{K}}$-class of $f$ in $P$. Recall that $\lambda_{i}=\left|A_{i}\right|$ for each $i \in \mathbf{r}$, and that $\Lambda_{I}=\lambda_{i_{1}} \cdots \lambda_{i_{m}}$ if $I=\left\{i_{1}, \ldots, i_{m}\right\} \subseteq \mathbf{r}$. If $Y$ is a set and $0 \leq m \leq|Y|$, we write $\left(\begin{array}{l}Y \\ m\end{array}\right)$ for the set of all $m$-element subsets of $Y$. Recall that a rectangular band is a semigroup of the form $I \times J$ with product $\left(i_{1}, j_{1}\right)\left(i_{2}, j_{2}\right)=\left(i_{1}, j_{2}\right)$, and that a rectangular group is a direct product of a rectangular band with a group.

Theorem 5.7. Let $f=\left(\begin{array}{ccc}F_{1} & \cdots & F_{m} \\ f_{1} & \cdots & f_{m}\end{array}\right) \in P$, where $m=\operatorname{rank}(f)$ and $f_{i} \in A_{k_{i}}$ for each $i$, and put $I=$ $\left\{k_{1}, \ldots, k_{m}\right\}$.

(i) $\widehat{R}_{f}$ is the union of $m^{n-r} \mathscr{R}^{a}$-classes of $P$.

(ii) $\widehat{L}_{f}$ is the union of $\Lambda_{I} \mathscr{L}^{a}$-classes of $P$.

(iii) $\widehat{H}_{f}$ is the union of $m^{n-r} \Lambda_{I} \mathscr{H}^{a}$-classes of $P$, each of which has size $m$ !. The map $\phi: P \rightarrow \mathcal{T}_{A}$ is injective when restricted to any $\mathscr{H}^{a}$-class of $P$.

(iv) If $H_{\bar{f}}$ is a non-group $\mathscr{H}$-class of $\mathcal{T}_{A}$, then each $\mathscr{H}^{a}$-class of $P$ contained in $\widehat{H}_{f}$ is a non-group.

(v) If $H_{\bar{f}}$ is a group $\mathscr{H}$-class of $\mathcal{T}_{A}$, then each $\mathscr{H}^{a}$-class of $P$ contained in $\widehat{H}_{f}$ is a group isomorphic to the symmetric group $\mathcal{S}_{m}$. Further, $\widehat{H}_{f}$ is a rectangular group; specifically, $\widehat{H}_{f}$ is isomorphic to a direct product of an $m^{n-r} \times \Lambda_{I}$ rectangular band with $\mathcal{S}_{m}$.

(vi) $\widehat{\mathscr{D}}=\mathscr{D}^{a}$, so $\widehat{D}_{f}=D_{f}^{a}=D_{m}^{a}=\{g \in P: \operatorname{rank}(g)=m\}$ is the union of:

(a) $m^{n-r} S(r, m) \mathscr{R}^{a}$-classes of $P$,

(b) $\sum_{J \in\left(\begin{array}{c}\mathbf{r} \\ m\end{array}\right)} \Lambda_{J} \mathscr{L}^{a}$-classes of $P$,

(c) $m^{n-r} S(r, m) \sum_{J \in\left(\begin{array}{c}\mathbf{r} \\ m\end{array}\right)} \Lambda_{J} \mathscr{H}^{a}$-classes of P.

Proof. First observe that if $\rho: S \rightarrow T$ is an epimorphism of semigroups, and if $K$ is a $\mathscr{K}$-class of $T$ where $\mathscr{K}$ is one of $\mathscr{L}, \mathscr{R}, \mathscr{H}$, then $K \rho^{-1}$ is a union of $\mathscr{K}$-classes of $S$.

(i) By the above observation, it suffices to count the number of $\mathscr{R}^{a}$-classes contained in $\widehat{R}_{f}$. An $\mathscr{R}^{a}$ class $R_{g}^{a}$ contained in $\widehat{R}_{f}$ is completely determined by the common kernel of all its members, namely $\operatorname{ker}(g)$. Such a kernel is constrained so that it has $m$ equivalence classes and $\operatorname{ker}(\bar{g})=\operatorname{ker}(\bar{f})=$ $\left(F_{1} \cap A|\cdots| F_{m} \cap A\right)$. To construct $\operatorname{ker}(g)$ from $\operatorname{ker}(\bar{g})$, the remaining $n-r$ elements of $X \backslash A$ may be assigned to the $m \operatorname{ker}(\bar{g})$-classes arbitrarily, and there are $m^{n-r}$ ways to do this.

(ii) An $\mathscr{L}^{a}$-class $L_{g}^{a}$ contained in $\widehat{L}_{f}$ is completely determined by the common image of all its members, namely $\operatorname{im}(g)$. Such an image is constrained so that it has size $m$ and $\operatorname{im}(\bar{g})=\operatorname{im}(\bar{f})=\left\{a_{k_{1}}, \ldots, a_{k_{m}}\right\}$. So $\operatorname{im}(g)$ must contain one element of $A_{k_{i}}$ for each $i$, and may be chosen in $\lambda_{k_{1}} \cdots \lambda_{k_{m}}=\Lambda_{I}$ ways. 
(iii) The statement concerning the number of $\mathscr{H}^{a}$-classes contained in $\widehat{H}_{f}$ follows immediately from (i) and (ii). By Theorem $4.2, H_{f}^{a}=H_{f}$, so $\left|H_{f}^{a}\right|=m$ !. If $g \mathscr{H}^{a} f$, then $g=\left(\begin{array}{ccc}F_{1} & \cdots & F_{m} \\ f_{1 q} & \cdots & f_{m q}\end{array}\right)$ and $\bar{g}=$ $\left(\begin{array}{cccc}F_{1} \cap A & \ldots & F_{m} \cap A \\ a_{k_{1 q}} & \ldots & a_{k_{m q}}\end{array}\right)$ for some $q \in \mathcal{S}_{m}$. So it follows that $\phi$ is injective when restricted to $H_{f}^{a}$. Since $H_{f}^{a}$ is an arbitrary $\mathscr{H}^{a}$-class of $\operatorname{Reg}\left(\mathcal{T}_{X}^{a}\right)$, the proof of (iii) is complete.

(iv) If $H_{\bar{f}}$ is a non-group $\mathscr{H}$-class, then $\bar{g}^{2} \notin H_{\bar{f}}$ for all $g \in \widehat{H}_{f}$. Since $\operatorname{im}\left(\bar{g}^{2}\right) \subseteq \operatorname{im}(\bar{g})$ and $\operatorname{ker}\left(\bar{g}^{2}\right) \supseteq \operatorname{ker}(\bar{g})$, it then follows that $\operatorname{rank}\left(g^{2}\right)=\operatorname{rank}\left(\bar{g}^{2}\right)<\operatorname{rank}(\bar{g})=\operatorname{rank}(g)$, so $g^{2} \notin H_{g}^{a}$, whence $H_{g}^{a}$ is a non-group $\mathscr{H}^{a}$-class of $P$.

(v) Suppose $H_{\bar{f}}$ is a group. Then $\bar{g}^{2} \in H_{\bar{f}}$ for any $g \in \widehat{H}_{f}$, so $\operatorname{rank}\left(g^{2}\right)=\operatorname{rank}\left(\bar{g}^{2}\right)=\operatorname{rank}(\bar{g})=\operatorname{rank}(g)$. But $\operatorname{im}\left(g^{2}\right) \subseteq \operatorname{im}(g)$ and $\operatorname{ker}\left(g^{2}\right) \supseteq \operatorname{ker}(g)$, so it follows that $\operatorname{im}\left(g^{2}\right)=\operatorname{im}(g)$ and $\operatorname{ker}\left(g^{2}\right)=\operatorname{ker}(g)$, whence $g^{2} \mathscr{H}^{a} g$, whence $H_{g}^{a}$ is a group. By (iii), the restriction of $\phi$ to $H_{f}^{a}$ yields an isomorphism onto $H_{\bar{f}} \cong \mathcal{S}_{m}$.

Consider an arbitrary element $g \in \widehat{H}_{f}$, and write $\operatorname{ker}(g)=\left(G_{1}|\cdots| G_{m}\right)$ and $\operatorname{im}(g)=\left\{g_{1}, \ldots, g_{m}\right\}$ where $a_{k_{i}} \in G_{i}$ and $g_{i} \in A_{k_{i}}$ for each $i \in \mathbf{m}$. Then there is a permutation $p_{g} \in \mathcal{S}_{m}$ such that $g=\left(\begin{array}{ccc}G_{1} & \cdots & G_{m} \\ g_{1 p_{g}} & \cdots & g_{m p_{g}}\end{array}\right)$. In this way, we see that $g$ is completely determined by $\operatorname{ker}(g), \operatorname{im}(g)$ and $p_{g}$, and we write $g \equiv\left[\operatorname{ker}(g), \operatorname{im}(g), p_{g}\right]$. If $h=\left(\begin{array}{ccc}H_{1} & \cdots & H_{m} \\ h_{1 p_{h}} & \cdots & h_{m p_{h}}\end{array}\right) \equiv\left[\operatorname{ker}(h), \operatorname{im}(h), p_{h}\right]$ is another element of $\widehat{H}_{f}$, then we have $g \star h=\left[\operatorname{ker}(g), \operatorname{im}(h), p_{g} p_{h}\right]$. Indeed, we have $\operatorname{ker}(g \star h)=\operatorname{ker}(g)$ and $\operatorname{im}(g \star h)=\operatorname{im}(h)$, as $g \star h \in \widehat{H}_{f}$ gives $\operatorname{rank}(g \star h)=m$, and if $x \in G_{i}$ is arbitrary, then

$$
x \stackrel{g}{\longmapsto} g_{i p_{g}} \stackrel{a}{\longmapsto} a_{k_{i p_{g}}} \stackrel{h}{\longmapsto} h_{i p_{g} p_{h}} .
$$

Now let $K=\left\{\operatorname{ker}(g): g \in \widehat{H}_{f}\right\}$ and $I=\left\{\operatorname{im}(g): g \in \widehat{H}_{f}\right\}$. Then $K \times I$ is a rectangular band under the product $(\beta, B)(\gamma, C)=(\beta, C)$, and by the above rule for multiplication in $\widehat{H}_{f}$, we immediately see that the map

$$
\widehat{H}_{f} \rightarrow K \times I \times \mathcal{S}_{m}: g \equiv\left[\operatorname{ker}(g), \operatorname{im}(g), p_{g}\right] \mapsto\left(\operatorname{ker}(g), \operatorname{im}(g), p_{g}\right)
$$

is an isomorphism. The dimensions of the rectangular band are given by parts (i) and (ii), above.

(vi) We deduce $\widehat{\mathscr{D}}=\mathscr{D}^{a}$ immediately from the fact that $\operatorname{rank}(\bar{f})=\operatorname{rank}(f)$ for all $f \in P$. The number of $\widehat{\mathscr{R}}$-classes in $D_{m}^{a}$ is equal to the number of $\mathscr{R}$-classes in $D_{m} \subseteq \mathcal{T}_{A}$, which is equal to $S(r, m)$; (a) now follows from (i). Part (b) follows from (ii) and the fact that the $\mathscr{L}$-classes contained in $D_{m} \subseteq \mathcal{T}_{A}$ (and hence the $\widehat{\mathscr{L}}$-classes contained in $D_{m}^{a}$ ) are indexed by the $m$-element subsets of $A$. Part (c) follows immediately from (a) and (b).

Remark 5.8. See also [70, Proposition 3.1] for formulae for the number of singleton $\mathscr{R}^{a}$ - and $\mathscr{L}^{a}$-classes of $\mathcal{T}_{X}^{a}$, and various other parameters.

As an immediate consequence of Theorem 5.7, we may give the size of $P=\operatorname{Reg}\left(\mathcal{T}_{X}^{a}\right)$.

Corollary 5.9. We have $\left|\operatorname{Reg}\left(\mathcal{T}_{X}^{a}\right)\right|=\sum_{m=1}^{r} m ! m^{n-r} S(r, m) \sum_{I \in\left(\begin{array}{c}\mathbf{r} \\ m\end{array}\right)} \Lambda_{I}$.

Proof. From parts (vi) and (iii) of Theorem 5.7, we have $\left|D_{m}^{a}\right|=m ! m^{n-r} S(r, m) \sum_{I \in\left(\begin{array}{c}\mathbf{r} \\ m\end{array}\right)} \Lambda_{I}$ for each $m \in \mathbf{r}$. Summing over all $m$ gives the result.

The top $\mathscr{D}^{a}$-class of $P$ is the set

$$
D_{r}^{a}=\mathcal{S}_{A} \phi^{-1}=\{f \in P: \operatorname{rank}(f)=r\} .
$$

We will write $D=D_{r}^{a}$ for this set. As a special case of Theorem 5.7(v), $D$ is a rectangular group; it is isomorphic to the direct product of an $r^{n-r} \times \Lambda$ rectangular band with the symmetric group $\mathcal{S}_{r}$. (Recall 
that $\Lambda=\lambda_{1} \cdots \lambda_{r}$.) Since $D$ is the pre-image of $\mathcal{S}_{A}$ under the map $\phi: P \rightarrow \mathcal{T}_{A}$, we may think of $D$ as a kind of "inflation" of $\mathcal{S}_{A}$, the group of units of $\mathcal{T}_{A}$. In fact, we will soon see that $D=\operatorname{RP}(P)$ is precisely the set of regularity preserving elements of $P$, so that $D$ may be thought of as an alternative to the group of units in the non-monoid $P$, as noted in Section 3 .

In order to avoid confusion when discussing idempotents, if $U \subseteq \mathcal{T}_{X}$, we will write

$$
E(U)=\{f \in U: f=f \circ f\} \quad \text { and } \quad E_{a}(U)=\{f \in U: f=f \star f\}
$$

for the set of idempotents from $U$ with respect to the different operations on $\mathcal{T}_{X}$ and $\mathcal{T}_{X}^{a}$. Recall that an element $u$ of a semigroup $S$ is a mididentity if $x u y=x y$ for all $x, y \in S$.

Lemma 5.10. Let $e \in E_{a}(D)$. Then aea $=a$. In particular, $e$ is a mididentity for both $\mathcal{T}_{X}^{a}$ and $P$.

Proof. Since $\operatorname{rank}(e)=r$, we may write $e=\left(\begin{array}{ccc}E_{1} & \ldots & E_{r} \\ e_{1} & \ldots & e_{r}\end{array}\right)$. Since $\alpha$ separates $\operatorname{im}(e)=\left\{e_{1}, \ldots, e_{r}\right\}$, we may assume (reordering if necessary) that $e_{i} \in A_{i}$ for each $i$. It follows that $e a=\left(\begin{array}{ccc}E_{1} & \cdots & E_{r} \\ a_{1} & \cdots & a_{r}\end{array}\right)$. Since $e \in E_{a}(D)$, we see that $e=e \star e=e a e$. It follows that $e a=e a e a$, so $e a \in E\left(\mathcal{T}_{X}\right)$, whence $a_{i} \in E_{i}$ for each $i$. It follows then that $a e a=\left(\begin{array}{lll}A_{1} & \ldots & A_{r} \\ a_{1} & \ldots & a_{r}\end{array}\right)=a$. If $f, g \in \mathcal{T}_{X}$, then $f \star e \star g=$ faeag $=f a g=f \star g$, showing that $e$ is a mididentity for $\mathcal{T}_{X}^{a}$ (and hence also for $P \subseteq \mathcal{T}_{X}^{a}$ ) and completing the proof.

Proposition 5.11. The top $\mathscr{D}^{a}$-class, $D=D_{r}^{a}$, of $P=\operatorname{Reg}\left(\mathcal{T}_{X}^{a}\right)$ is precisely the set $\operatorname{RP}(P)$ of all regularity preserving elements of $P$.

Proof. By Proposition 3.5(i), it suffices to show that $E_{a}(\mathrm{RP}(P))=E_{a}(D)$. By Proposition 3.5(iii) and Lemma 5.10 , we see that $E_{a}(D) \subseteq E_{a}(\mathrm{RP}(P))$. Conversely, suppose $e \in E_{a}(P) \backslash E_{a}(D)$. Then $\operatorname{rank}(e)<r$, and so if $f \in E_{a}(D)$ is arbitrary, then $\operatorname{rank}(f \star e)=\operatorname{rank}(f a e) \leq \operatorname{rank}(e)<r$, so $f \star e$ does not belong to $D=D_{f}^{a}$ and, in particular, $f \star e$ is not $\mathscr{R}^{a}$-related to $f$, from which we deduce from Proposition 3.5(ii) that $e \notin \mathrm{RP}(P)$. This shows that $E_{a}(\mathrm{RP}(P)) \subseteq E_{a}(D)$, and completes the proof.

Our next goal is to calculate the rank of $P=\operatorname{Reg}\left(\mathcal{T}_{X}^{a}\right)$. Recall that the relative rank, denoted $\operatorname{rank}(S: U)$, of a semigroup $S$ with respect to a subset $U \subseteq S$ is defined to be the minimum cardinality of a subset $V \subseteq S$ such that $S=\langle U \cup V\rangle$. The concept of relative rank was first introduced in [45], and has played a major role in a number of investigations $[2,11,34,36,38]$.

Lemma 5.12. We have $\operatorname{rank}(P)=\operatorname{rank}(D)+\operatorname{rank}(P: D)$.

Proof. This follows quickly from the fact that $D$ is a subsemigroup of $P$ and $P \backslash D$ an ideal.

The next result may be easily be proved directly, but it is a special case of [61, Theorem 4.7] (see also [31]) so we omit the proof.

Lemma 5.13 (Ruškuc [61]). Let $I$ and $J$ be non-empty sets, and $G$ a group. Let $S=I \times J \times G$ be the rectangular group with product defined by $\left(i_{1}, j_{1}, g_{1}\right)\left(i_{2}, j_{2}, g_{2}\right)=\left(i_{1}, j_{2}, g_{1} g_{2}\right)$. Then

$$
\operatorname{rank}(S)=\max \{|I|,|J|, \operatorname{rank}(G)\} .
$$

We wish to apply Lemma 5.13 to calculate the rank of the rectangular group $D$. To do this, we need to calculate $\max \left\{r^{n-r}, \Lambda\right\}$. Recall that we are assuming $1<r<n$.

Lemma 5.14. We have $r^{n-r} \geq \Lambda=\lambda_{1} \cdots \lambda_{r}$.

Proof. First note that if $r=2$ and $n=3$, then we must have $\left\{\lambda_{1}, \lambda_{2}\right\}=\{1,2\}$, in which case $r^{n-r}=\Lambda=2$. Now suppose $(r, n) \neq(2,3)$. Elementary calculus shows that the maximum value of the product $x_{1} \cdots x_{r}$, where $x_{1}+\cdots+x_{r}=n$ and $x_{1}, \ldots, x_{r} \geq 0$ are real numbers, occurs when $x_{1}=\cdots=x_{r}=n / r$. It 
follows that $\Lambda \leq(n / r)^{r}=n^{r} / r^{r}$. So it suffices to show that $n^{r} / r^{r} \leq r^{n-r}=r^{n} / r^{r}$, which is equivalent to $n^{r} \leq r^{n}$. This, in turn, is equivalent to $\ln (n) / n \leq \ln (r) / r$. Now, $f(x)=\ln (x) / x$ is a decreasing function for $x>e \approx 2.718$. In particular, $f(3)>f(4)>f(5)>\cdots$, so the result holds for $r \geq 3$. We also have $f(2)=f(4)$, so the result holds for $r=2$ and $n \geq 4$. We have already covered the case $(r, n)=(2,3)$.

Corollary 5.15. We have $\operatorname{rank}(D)=r^{n-r}$.

Proof. Recall that $D$ is isomorphic to the direct product of an $r^{n-r} \times \Lambda$ rectangular band with the symmetric group $\mathcal{S}_{r}$. So Lemma 5.13 gives $\operatorname{rank}(D)=\max \left\{r^{n-r}, \Lambda, \operatorname{rank}\left(\mathcal{S}_{r}\right)\right\}$. We have already seen that $r^{n-r} \geq \Lambda$. Also, $\operatorname{rank}\left(\mathcal{S}_{2}\right)=1$, while $\operatorname{rank}\left(\mathcal{S}_{r}\right)=2$ if $r \geq 3$. So it follows that $r^{n-r} \geq \operatorname{rank}\left(\mathcal{S}_{r}\right)$.

The next technical lemma will help us calculate $\operatorname{rank}(P: D)$. It is quite a bit stronger than we need at this point (we only require the $m=r$ case at the moment), but we will use the full strength in subsequent sections when we consider ideals and the idempotent generated subsemigroup of $\mathcal{T}_{X}^{a}$.

Lemma 5.16. Suppose $f, g \in P$ are such that $\bar{f}=\bar{g}$. Then for any $\operatorname{rank}(f) \leq m \leq r$, there exist idempotents $e_{1}, e_{2} \in E_{a}\left(D_{m}^{a}\right)$ such that $f=e_{1} \star g \star e_{2}$.

Proof. Put $l=\operatorname{rank}(f)=\operatorname{rank}(g)$ and write $f=\left(\begin{array}{ccc}F_{1} & \ldots & F_{l} \\ f_{1} & \ldots & f_{l}\end{array}\right)$ and $g=\left(\begin{array}{ccc}G_{1} & \ldots & G_{l} \\ g_{1} & \ldots & g_{l}\end{array}\right)$, where $f_{i} \in A_{k_{i}}$ for each $i$. Since $\left(\begin{array}{cccc}F_{1} \cap A & \ldots & F_{l} \cap A \\ a_{k_{1}} & \ldots & a_{k_{l}}\end{array}\right)=\bar{f}=\bar{g}$, we may assume (reordering if necessary) that $g_{i} \in A_{k_{i}}$ for all $i$, in which case also $G_{i} \cap A=F_{i} \cap A$. Let $\mathbf{r} \backslash\left\{k_{1}, \ldots, k_{l}\right\}=\left\{j_{1}, \ldots, j_{r-l}\right\}$, and put $B=A_{j_{m-l+1}} \cup \cdots \cup A_{j_{r-l}}$. (Note that $B=\emptyset$ if $m=r$.) Define

$$
e_{2}=\left(\begin{array}{ccccccc}
A_{k_{1}} \cup B & A_{k_{2}} & \cdots & A_{k_{l}} & A_{j_{1}} & \cdots & A_{j_{m-l}} \\
f_{1} & f_{2} & \cdots & f_{l} & a_{j_{1}} & \cdots & a_{j_{m-l}}
\end{array}\right) .
$$

For each $s \in 1$, let $F_{s} \cap A=\left\{a_{i_{s 1}}, \ldots, a_{i_{s q_{s}}}\right\}$, noting that $F_{s} \cap A \neq \emptyset$ and $q_{1}+\cdots+q_{l}=r$. For each $s$, choose $1 \leq p_{s} \leq q_{s}$ such that $p_{1}+\cdots+p_{l}=m$, and choose a partition $F_{s}=F_{s 1} \sqcup \cdots \sqcup F_{s p_{s}}$ so that $a_{i_{s t}} \in F_{s t}$ for each $t$. Define

$$
e_{1}=\left(\begin{array}{ccccccc}
F_{11} & \cdots & F_{1 p_{1}} & \cdots & F_{l 1} & \cdots & F_{l_{p}} \\
a_{i_{11}} & \cdots & a_{i_{1 p_{1}}} & \cdots & a_{i_{11}} & \cdots & a_{i_{l_{l}}}
\end{array}\right) \text {. }
$$

One may easily check that $e_{1}, e_{2} \in E\left(\mathcal{T}_{X}\right)$. Since also $e_{1} a=e_{1}$ and $a e_{2}=e_{2}$, it follows that $e_{1}, e_{2} \in E_{a}\left(D_{m}^{a}\right)$. Now let $x \in F_{s}$ be arbitrary. Then $x e_{1} \in F_{s} \cap A=G_{s} \cap A$, so

$$
x \stackrel{e_{1}}{\longmapsto} x e_{1} \stackrel{a}{\longmapsto} x e_{1} \stackrel{g}{\longmapsto} g_{s} \stackrel{a}{\longmapsto} a_{k_{s}} \stackrel{e_{2}}{\longmapsto} f_{s}=x f,
$$

showing that $f=e_{1} \star g \star e_{2}$, as desired.

Lemma 5.17. If $f \in D_{r-1}^{a}$ is arbitrary, then $P=\langle D \cup\{f\}\rangle_{a}$. Consequently, $\operatorname{rank}(P: D)=1$.

Proof. Since $\langle D\rangle_{a}=D \neq P$ (as $\left.r>1\right), \operatorname{rank}(P: D) \geq 1$ so it suffices to prove the first statement. Note that $\bar{D}=\{\bar{g}: g \in D\}$ is equal to $\mathcal{S}_{A}$, and $\bar{f} \in \mathcal{T}_{A}$ satisfies $\operatorname{rank}(\bar{f})=r-1$. It follows that $\mathcal{T}_{A}=\langle\bar{D} \cup\{\bar{f}\}\rangle$. Now let $g \in P$ be arbitrary. Choose $h_{1}, \ldots, h_{k} \in D \cup\{f\}$ such that $\bar{g}=\bar{h}_{1} \cdots \bar{h}_{k}$, and put $h=h_{1} \star \cdots \star h_{k} \in\langle D \cup\{f\}\rangle_{a}$. Then $\bar{h}=\bar{g}$, so Lemma 5.16 tells us that $g=e_{1} \star h \star e_{2} \in\langle D \cup\{f\}\rangle_{a}$ for some $e_{1}, e_{2} \in E_{a}(D)$.

As an immediate consequence of Lemmas 5.12 and 5.17 and Corollary 5.15, we have the following.

Theorem 5.18. If $1<r<n$, then $\operatorname{rank}\left(\operatorname{Reg}\left(\mathcal{T}_{X}^{a}\right)\right)=r^{n-r}+1$.

Remark 5.19. It was shown in [64, Theorem 3.6] that $\operatorname{rank}(\operatorname{Reg}(\mathcal{T}(X, A)))=r^{n-r}+1$, also. See also Theorem 7.4 and Remark 7.5. If $r=1$, then $\operatorname{Reg}\left(\mathcal{T}_{X}^{a}\right)=D_{1}$ is an $n$-element right zero semigroup, so we have $\operatorname{rank}\left(\operatorname{Reg}\left(\mathcal{T}_{X}^{a}\right)\right)=n$ in this case. If $r=n$, then $\operatorname{Reg}\left(\mathcal{T}_{X}^{a}\right)=\mathcal{T}_{X}^{a} \cong \mathcal{T}_{X}$, so $\operatorname{rank}\left(\operatorname{Reg}\left(\mathcal{T}_{X}^{a}\right)\right)=\operatorname{rank}\left(\mathcal{T}_{X}\right)$, which is equal to 1 (if $n \leq 1$ ), 2 (if $n=2$ ) or 3 (if $n \geq 3$ ).

Remark 5.20. The natural task of classifying and enumerating the generating sets of $P$ of the minimal size $r^{n-r}+1$ seems virtually unassailable. Indeed, by the proof of Lemma 5.13 (see 61, Theorem 4.7]), such a classification would involve classifying and enumerating all generating sets of $\mathcal{S}_{r}$ of size at most $r^{n-r}$. 


\section{The idempotent generated subsemigroup $\left\langle E_{a}\left(\mathcal{T}_{X}^{a}\right)\right\rangle_{a}$}

In this section, we investigate the idempotent generated subsemigroup $\mathcal{E}_{X}^{a}=\left\langle E_{a}\left(\mathcal{T}_{X}^{a}\right)\right\rangle_{a}$ of $\mathcal{T}_{X}^{a}$. Our main results include a proof that $\mathcal{E}_{X}^{a}=E_{a}(D) \cup(P \backslash D)$, a calculation of $\operatorname{rank}\left(\mathcal{E}_{X}^{a}\right)=\operatorname{idrank}\left(\mathcal{E}_{X}^{a}\right)$, and an enumeration of the idempotent generating sets of this minimal possible size. Since the solution to every problem we consider is trivial when $r=1$, and well-known when $r=n$, we will continue to assume that $1<r<n$. To simplify notation, we will write $E=E_{a}\left(\mathcal{T}_{X}^{a}\right)=E_{a}(P)$, so $\mathcal{E}_{X}^{a}=\langle E\rangle_{a}$. We begin with a simple observation; part (ii) is proved in [25, Proposition 13.3.2], where the idempotents were characterised in a different way (we include a short proof for completeness).

Proposition 6.1. (i) $E=E_{a}\left(\mathcal{T}_{X}^{a}\right)=\left\{f \in \mathcal{T}_{X}:\left.(a f)\right|_{\operatorname{im}(f)}=\mathrm{id}_{\mathrm{im}(f)}\right\}$;

(ii) $|E|=\sum_{m=1}^{r} m^{n-m} \sum_{I \in\left(\begin{array}{c}\mathbf{r} \\ m\end{array}\right)} \Lambda_{I}$.

Proof. Part (i) is easily checked. For part (ii), note that to specify an idempotent $f \in E$, we first choose $m=\operatorname{rank}(f)=\operatorname{rank}(\bar{f}) \in \mathbf{r}$, then $\operatorname{im}(\bar{f})=\left\{a_{i_{1}}, \ldots, a_{i_{m}}\right\}$, then $\operatorname{im}(f)=\left\{b_{1}, \ldots, b_{m}\right\}$ where $b_{k} \in A_{i_{k}}$ for each $k \in \mathbf{m}$. Note that the condition $\left.(a f)\right|_{\operatorname{im}(f)}=\operatorname{id}_{\operatorname{im}(f)}$ simply says that $a_{i_{k}} f=b_{k}$ for each $k$. The remaining $n-m$ points of $X \backslash\left\{a_{i_{1}}, \ldots, a_{i_{m}}\right\}$ may be mapped arbitrarily by $f$ to any of the points from $\left\{b_{1}, \ldots, b_{m}\right\}$. Multiplying the number of choices at each step, and adding as appropriate, gives the desired result.

Lemma 6.2. If $f \in E\left(\mathcal{T}_{A}\right)$, then there exists $e \in E=E_{a}\left(\mathcal{T}_{X}^{a}\right)$ such that $f=\bar{e}$ and $\operatorname{rank}(e)=\operatorname{rank}(f)$.

Proof. One easily checks that $e=\left(\begin{array}{ccc}A_{1} & \cdots & A_{r} \\ a_{1} f & \cdots & a_{r} f\end{array}\right)$ satisfies the desired conditions.

Recall that $\mathcal{T}_{A} \backslash \mathcal{S}_{A}$ is idempotent generated; see Theorem 2.2 .

Lemma 6.3. Let $V \subseteq E_{a}(P \backslash D)$ be an arbitrary set of idempotents such that $\mathcal{T}_{A} \backslash \mathcal{S}_{A}=\langle\bar{V}\rangle$. Then $\left\langle E_{a}(D) \cup V\right\rangle_{a}$ contains $P \backslash D$.

Proof. Let $f \in P \backslash D$ be arbitrary. Choose $e_{1}, \ldots, e_{k} \in V$ so that $\bar{f}=\bar{e}_{1} \cdots \bar{e}_{k}$, and put $g=e_{1} \star \cdots \star e_{k} \in\langle V\rangle_{a}$. So $\bar{g}=\bar{f}$, and Lemma 5.16 tells us that there exist $e_{0}, e_{k+1} \in E_{a}(D)$ such that $f=e_{0} \star g \star e_{k+1} \in$ $\left\langle E_{a}(D) \cup V\right\rangle_{a}$.

We may now describe the idempotent generated subsemigroup $\mathcal{E}_{X}^{a}=\left\langle E_{a}\left(\mathcal{T}_{X}^{a}\right)\right\rangle_{a}$ of $\mathcal{T}_{X}^{a}$.

Theorem 6.4. We have $\mathcal{E}_{X}^{a}=\langle E\rangle_{a}=E_{a}(D) \cup(P \backslash D)$, where $E=E_{a}\left(\mathcal{T}_{X}^{a}\right)=E_{a}(P)$ and $D=D_{r}^{a}$ is the top $\mathscr{D}^{a}$-class of $P=\operatorname{Reg}\left(\mathcal{T}_{X}^{a}\right)$.

Proof. First, $E_{a}(D) \subseteq E$, and it follows from Lemma 6.3 that $P \backslash D \subseteq \mathcal{E}_{X}^{a}$. It remains to show that $\mathcal{E}_{X}^{a} \subseteq E_{a}(D) \cup(P \backslash D)$. So suppose $f \in \mathcal{E}_{X}^{a}$, and consider an expression $f=e_{1} \star \cdots \star e_{k}$, where $e_{1}, \ldots, e_{k} \in E$. We must show that $f \in E_{a}(D) \cup(P \backslash D)$. If $f \in P \backslash D$, we are done, so suppose $f \in D$. Since $P \backslash D$ is an ideal, it follows that $e_{1}, \ldots, e_{k} \in D$. But $D$ is a rectangular group, so $E_{a}(D)$ is a rectangular band. In particular, $f=e_{1} \star \cdots \star e_{k} \in E_{a}(D)$.

Remark 6.5. Theorem 6.4 is a pleasing analogue of Howie's result 39 that $\left\langle E\left(\mathcal{T}_{X}\right)\right\rangle=\{1\} \cup\left(\mathcal{T}_{X} \backslash \mathcal{S}_{X}\right)$, since $\{1\}=E\left(\mathcal{S}_{X}\right)$, where $\mathcal{S}_{X}$ is the top $\mathscr{D}$-class of $\mathcal{T}_{X}$. Also, $\mathcal{S}_{X}=G\left(\mathcal{T}_{X}\right)=\operatorname{RP}\left(\mathcal{T}_{X}\right)$ and, while $\mathcal{T}_{X}^{a}$ has no group of units as it is not a monoid, it is still the case that $D=\operatorname{RP}(P)$.

Now that we have described the elements of the semigroup $\mathcal{E}_{X}^{a}$, the next natural task is to calculate its rank and idempotent rank. To do this, we need the first part of the next result; the second part will be of use when we later enumerate the idempotent generating sets of $\mathcal{E}_{X}^{a}$ of minimal possible size. 
Lemma 6.6. Let $I$ and $J$ be non-empty sets, and $S=I \times J$ the rectangular band with product defined by $\left(i_{1}, j_{1}\right)\left(i_{2}, j_{2}\right)=\left(i_{1}, j_{2}\right)$. Then

$$
\operatorname{rank}(S)=\operatorname{idrank}(S)=\max \{|I|,|J|\} .
$$

If $I$ and $J$ are finite, then the number of (idempotent) generating sets of this smallest possible size is equal to $y ! S(x, y)$, where $x=\max \{|I|,|J|\}$ and $y=\min \{|I|,|J|\}$.

Proof. Note that $S$ is (isomorphic to) a rectangular group with respect to a trivial group, which has rank 1, so the statement about $\operatorname{rank}(S)=\operatorname{idrank}(S)$ follows immediately from Lemma 5.13 (or may easily be proved directly). Now let $U$ be an arbitrary generating set of $S$ of minimal possible size. By duality, we may assume that $x=|I|$ and $y=|J|$. By considering an expression $(i, j)=u_{1} \cdots u_{k}$, where $u_{1}, \ldots, u_{k} \in U$, we see that for each $i \in I, U$ contains $\left(i, j_{i}\right)$ for some $j_{i} \in J$. Since we are assuming that $|U|=x=|I|$, we see that in fact $U=\left\{\left(i, j_{i}\right): i \in I\right\}$. A similar consideration shows that $J=\left\{j_{i}: i \in I\right\}$, so $i \mapsto j_{i}$ defines a surjective map $I \rightarrow J$. (In fact, considered as a binary relation, $U$ is a surjective map $I \rightarrow J$.) Conversely, any surjective map $I \rightarrow J$ determines an idempotent generating set of $S$ of size $x=|I|$. Since the number of surjective functions from an $x$-set to a $y$-set is $y ! S(x, y)$, the result follows.

Since $E_{a}(D)$ is an $r^{n-r} \times \Lambda$ rectangular band, the next result follows from Lemmas 5.14 and 6.6 .

Corollary 6.7. We have $\operatorname{rank}\left(E_{a}(D)\right)=\operatorname{idrank}\left(E_{a}(D)\right)=r^{n-r}$, and the number of minimal (idempotent) generating sets of $E_{a}(D)$ is equal to $\Lambda ! S\left(r^{n-r}, \Lambda\right)$.

Theorem 6.8. We have $\operatorname{rank}\left(\mathcal{E}_{X}^{a}\right)=\operatorname{idrank}\left(\mathcal{E}_{X}^{a}\right)=r^{n-r}+\rho_{r}$, where $\rho_{2}=2$ and $\rho_{r}=\left(\begin{array}{l}r \\ 2\end{array}\right)$ if $r \geq 3$.

Proof. As in Lemma 5.12, we have $\operatorname{rank}\left(\mathcal{E}_{X}^{a}\right)=\operatorname{rank}\left(E_{a}(D)\right)+\operatorname{rank}\left(\mathcal{E}_{X}^{a}: E_{a}(D)\right)$ so, by Corollary 6.7, it remains to show that:

(i) there exists a set $V \subseteq E$ of size $\rho_{r}$ such that $\mathcal{E}_{X}^{a}=\left\langle E_{a}(D) \cup V\right\rangle_{a}$, and

(ii) if $W \subseteq \mathcal{E}_{X}^{a} \backslash E_{a}(D)=P \backslash D$ satisfies $\mathcal{E}_{X}^{a}=\left\langle E_{a}(D) \cup W\right\rangle_{a}$, then $|W| \geq \rho_{r}$.

Let $U \subseteq E\left(\mathcal{T}_{A}\right)$ be an arbitrary idempotent generating set of $\mathcal{T}_{A} \backslash \mathcal{S}_{A}$ with $|U|=\rho_{r}$. By Lemma 6.2, we may choose $V \subseteq E$ such that $|V|=\rho_{r}$ and $\bar{V}=U$. Since $U$ is a generating set of $\mathcal{T}_{A} \backslash \mathcal{S}_{A}$, Lemma 6.3 and Theorem 6.4 give $\left\langle E_{a}(D) \cup V\right\rangle_{a}=\mathcal{E}_{X}^{a}$, establishing (i).

Next, suppose $\mathcal{E}_{X}^{a}=\left\langle E_{a}(D) \cup W\right\rangle_{a}$, where $W \subseteq P \backslash D$. We will show that $\bar{W}$ generates $\mathcal{T}_{A} \backslash \mathcal{S}_{A}$. Indeed, let $g \in \mathcal{T}_{A} \backslash \mathcal{S}_{A}$ be arbitrary, and choose any $h \in P$ such that $\bar{h}=g$. Since $\operatorname{rank}(h)=\operatorname{rank}(\bar{h})=\operatorname{rank}(g)<r$, it follows that $h \in P \backslash D \subseteq \mathcal{E}_{X}^{a}$. Consider an expression $h=u_{1} \star \cdots \star u_{k}$, where $u_{1}, \ldots, u_{k} \in E_{a}(D) \cup W$. Now, $g=\bar{h}=\bar{u}_{1} \cdots \bar{u}_{k}$. If any of the $u_{i}$ belongs to $E_{a}(D)$, then $\bar{u}_{i}=1$, the identity element of $\mathcal{T}_{A}$. So the factor $\bar{u}_{i}$ is not needed in the product $g=\bar{u}_{1} \cdots \bar{u}_{k}$. After cancelling all such factors, we see that $g$ is a product of elements from $\bar{W}$. Since $g \in \mathcal{T}_{A} \backslash \mathcal{S}_{A}$ was arbitrary, we conclude that $\mathcal{T}_{A} \backslash \mathcal{S}_{A}=\langle\bar{W}\rangle$. In particular, $|W| \geq|\bar{W}| \geq \operatorname{rank}\left(\mathcal{T}_{A} \backslash \mathcal{S}_{A}\right)=\rho_{r}$, giving (ii).

Now that we know the size of a minimal (idempotent) generating set for $\mathcal{E}_{X}^{a}$, our next task is to enumerate the idempotent generating sets of this size. For $i, j \in \mathbf{r}$ with $i \neq j$, let $e_{i j} \in E\left(\mathcal{T}_{r}\right)$ and $\varepsilon_{i j} \in E\left(\mathcal{T}_{A}\right)$ be the transformations of $\mathbf{r}$ and $A$ (respectively) defined by

$$
k e_{i j}=\left\{\begin{array}{ll}
i & \text { if } k=j \\
k & \text { if } k \in \mathbf{r} \backslash\{j\}
\end{array} \quad \text { and } \quad a_{k} \varepsilon_{i j}= \begin{cases}a_{i} & \text { if } k=j \\
a_{k} & \text { if } k \in \mathbf{r} \backslash\{j\} .\end{cases}\right.
$$

Note that $a_{k} \varepsilon_{i j}=a_{k e_{i j}}$ for all $i, j, k$. Recall that $\mathbb{T}_{Y}$ denotes the set of all strongly connected tournaments on the vertex set $Y$ with $|Y| \geq 3$. We will write $\mathbb{T}_{r}$ for $\mathbb{T}_{\mathbf{r}}$. Recall also the convention that $\mathbb{T}_{2}=\mathbb{T}_{\mathbf{2}}$ consists of the single directed graph on vertex set $\mathbf{2}=\{1,2\}$ with edges $(1,2)$ and $(2,1)$. If $j \in \mathbf{r}$ and $\Gamma \in \mathbb{T}_{r}$, we write $d_{\Gamma}^{+}(j)$ for the in-degree of vertex $j$ in $\Gamma$. 
Theorem 6.9. The number of idempotent generating sets of $\mathcal{E}_{X}^{a}$ of the minimal possible size $r^{n-r}+\rho_{r}$ is equal to

$$
\left[(r-1)^{n-r} \Lambda\right]^{\rho_{r}} \Lambda ! S\left(r^{n-r}, \Lambda\right) \sum_{\Gamma \in \mathbb{T}_{r}} \frac{1}{\lambda_{1}^{d_{\Gamma}^{+}(1)} \cdots \lambda_{r}^{d_{\Gamma}^{+}(r)}} .
$$

Proof. Let $U$ be an arbitrary minimal idempotent generating set of $\mathcal{E}_{X}^{a}=E_{a}(D) \cup(P \backslash D)$. Put $U_{1}=$ $U \cap E_{a}(D)$ and $U_{2}=U \cap(P \backslash D)$. Since $P \backslash D$ is an ideal of $\mathcal{E}_{X}^{a}$, it follows that $U_{1}$ is a (minimal) idempotent generating set of $E_{a}(D)$. So, by Corollary 6.7, there are

$$
\Lambda ! S\left(r^{n-r}, \Lambda\right)
$$

choices for $U_{1}$. We multiply this by the number of choices for $U_{2}$. By the proof of Theorem $6.8, \bar{U}_{2}$ is a generating set of $\mathcal{T}_{A} \backslash \mathcal{S}_{A}$. Also, since $\left|\bar{U}_{2}\right| \leq\left|U_{2}\right|=|U|-\left|U_{1}\right|=\rho_{r}=\operatorname{idrank}\left(\mathcal{T}_{A} \backslash \mathcal{S}_{A}\right)$, it follows that $\bar{U}_{2}$ is a minimal idempotent generating set of $\mathcal{T}_{A} \backslash \mathcal{S}_{A}$, and therefore corresponds to a unique graph $\Gamma \in \mathbb{T}_{r}$. We will count the number of ways to choose $U_{2}$ so that $\bar{U}_{2}$ corresponds to $\Gamma$. Consider an edge $(i, j)$ in $\Gamma$. Then $\varepsilon_{i j} \in \bar{U}_{2}$, so there is a unique idempotent $\eta_{i j} \in U_{2}$ with $\varepsilon_{i j}=\bar{\eta}_{i j}$. To specify $\eta_{i j}$, we first choose $\operatorname{im}\left(\eta_{i j}\right)=\left\{b_{1}, \ldots, b_{j-1}, b_{j+1}, \ldots, b_{r}\right\}$, where $b_{k} \in A_{k}$ for each $k$. There are $\lambda_{1} \cdots \lambda_{j-1} \lambda_{j+1} \cdots \lambda_{r}=\Lambda / \lambda_{j}$ choices for $\operatorname{im}\left(\eta_{i j}\right)$. Once $\operatorname{im}\left(\eta_{i j}\right)$ is chosen, $\eta_{i j}$ is restricted by the fact that $a_{k} \eta_{i j}=b_{k e_{i j}}$ for each $k$. But the remaining $n-r$ elements of $X \backslash A$ may be mapped by $\eta_{i j}$ arbitrarily into the $r-1$ elements of $\operatorname{im}\left(\eta_{i j}\right)$, and there are $(r-1)^{n-r}$ ways to make these choices. So the total number of choices for $\eta_{i j}$ is equal to $(r-1)^{n-r} \Lambda / \lambda_{j}$. Since this value depends only on $j$, and since there are $d_{\Gamma}^{+}(j)$ edges of the form $(i, j)$, taking the product over all edges of $\Gamma$ gives a total of

$$
\prod_{j \in \mathbf{r}}\left[(r-1)^{n-r} \frac{\Lambda}{\lambda_{j}}\right]^{d_{\Gamma}^{+}(j)}=\left[(r-1)^{n-r} \Lambda\right]^{\rho_{r}} \frac{1}{\lambda_{1}^{d_{\Gamma}^{+}(1)} \cdots \lambda_{r}^{d_{\Gamma}^{+}(r)}}
$$

choices for $U_{2}$ with $\bar{U}_{2}$ corresponding to $\Gamma$ (noting that $\sum_{j \in \mathbf{r}} d_{\Gamma}^{+}(j)=\rho_{r}$ ). Summing 6.92) over all $\Gamma \in \mathbb{T}_{r}$ and multiplying by 6.9 .1 gives the result.

Remark 6.10. Theorem 6.9 is also valid if $r=n$, giving $\left|\mathbb{T}_{n}\right|$ generating sets for $\mathcal{E}_{X}=\left\langle E\left(\mathcal{T}_{X}\right)\right\rangle$ of size $1+\rho_{n}$, in agreement with Theorem 2.3. When $r=2$, the given expression reduces to $\Lambda^{2} \Lambda ! S\left(2^{n-2}, \Lambda\right)$.

\section{Ideals of $\operatorname{Reg}\left(\mathcal{T}_{X}^{a}\right)$}

In this final section, we consider the ideals of $P=\operatorname{Reg}\left(\mathcal{T}_{X}^{a}\right)$. In particular, we show that each of the proper ideals is idempotent generated, and we calculate the rank and idempotent rank, showing that these are equal. Again, the problems of this section have been solved in the case $r=n$ and are trivial if $r=1$, so we continue to assume that $1<r<n$. We first state the corresponding result for full transformation semigroups; for convenience, we state it in the context of $\mathcal{T}_{A}$.

Theorem 7.1 (Howie and McFadden [44]). The ideals of $\mathcal{T}_{A}$ are precisely the sets

$$
I_{m}=\bigcup_{j \in \mathbf{m}} D_{j}=\left\{f \in \mathcal{T}_{A}: \operatorname{rank}(f) \leq m\right\} \quad \text { for } 1 \leq m \leq r,
$$

and they form a chain: $I_{1} \subseteq \cdots \subseteq I_{r}$. If $m<r$, then $I_{m}=\left\langle E\left(D_{m}\right)\right\rangle$ is generated by the idempotents in its top $\mathscr{D}$-class, and

$$
\operatorname{rank}\left(I_{m}\right)=\operatorname{idrank}\left(I_{m}\right)= \begin{cases}S(r, m) & \text { if } 1<m<r \\ r & \text { if } m=1 .\end{cases}
$$

The next result is a strengthening Lemma 5.14 . 
Lemma 7.2. If $2 \leq m \leq r$, then

(i) $m^{n-r} \geq \Lambda_{I}$ for all $I \in\left(\begin{array}{c}\mathbf{r} \\ m\end{array}\right)$,

(ii) $m^{n-r} S(r, m) \geq \sum_{I \in\left(\begin{array}{c}\mathbf{r} \\ m\end{array}\right)} \Lambda_{I}$.

Proof. Let $I \in\left(\begin{array}{c}\mathbf{r} \\ m\end{array}\right)$. Since $\lambda_{j} \geq 1$ for all $j \in \mathbf{r} \backslash I, \sum_{i \in I} \lambda_{i} \leq n-r+m$. As in the proof of Lemma 5.14 . we deduce that $\Lambda_{I} \leq(n-r+m)^{m} / m^{m}$. So it suffices to prove that $(n-r+m)^{m} / m^{m} \leq m^{n-r}$, which is equivalent to

$$
(n-r+m)^{m} \leq m^{n-r+m} .
$$

Note that $n-r+m>m$, so again, as in the proof of Lemma 5.14, (7.2.1) is true unless $n-r+m=3$ and $m=2$. But in this exceptional case, we have $r=n-1$ and $m=2$ so that, without loss of generality, $\left(\lambda_{1}, \ldots, \lambda_{r}\right)=(2,1, \ldots, 1)$, giving $\Lambda_{I} \leq 2=m^{n-r}$. This completes the proof of (i). For (ii), we have

$$
\sum_{I \in\left(\begin{array}{c}
\mathbf{r} \\
m
\end{array}\right)} \Lambda_{I} \leq\left(\begin{array}{c}
r \\
m
\end{array}\right) m^{n-r} \leq S(r, m) m^{n-r},
$$

where we have used (i) and the fact that $S(r, m) \geq\left(\begin{array}{c}r \\ m\end{array}\right)$.

Remark 7.3. It follows from Theorem 5.7 and Lemma 7.2 that each $\widehat{\mathscr{H}}$ - and $\widehat{\mathscr{D}}=\mathscr{D}^{a}$-class of $P=\operatorname{Reg}\left(\mathcal{T}_{X}^{a}\right)$ not contained in $D_{1}^{a}$ is at least as "tall" as it is "wide"; that is, if $C$ is such a class, then $\left|C / \mathscr{R}^{a}\right| \geq\left|C / \mathscr{L}^{a}\right|$.

Theorem 7.4. The ideals of $P=\operatorname{Reg}\left(\mathcal{T}_{X}^{a}\right)$ are precisely the sets

$$
I_{m}^{a}=\bigcup_{j \in \mathbf{m}} D_{j}^{a}=\{f \in P: \operatorname{rank}(f) \leq m\} \quad \text { for } 1 \leq m \leq r,
$$

and they form a chain: $I_{1}^{a} \subseteq \cdots \subseteq I_{r}^{a}$. If $m<r$, then $I_{m}^{a}=\left\langle E_{a}\left(D_{m}^{a}\right)\right\rangle_{a}$ is generated by the idempotents in its top $\mathscr{D}^{a}$-class, and

$$
\operatorname{rank}\left(I_{m}^{a}\right)=\operatorname{idrank}\left(I_{m}^{a}\right)= \begin{cases}m^{n-r} S(r, m) & \text { if } 1<m<r \\ n & \text { if } m=1 .\end{cases}
$$

Proof. More generally, it may easily be checked that if the $\mathscr{J}$-classes of a semigroup $S$ form a chain, $J_{1}<\cdots<J_{q}$, then the ideals of $S$ are precisely the sets $I_{p}=J_{1} \cup \cdots \cup J_{p}$ for $1 \leq p \leq q$. Now suppose $m<r$, and let $f \in I_{m}^{a}$ be arbitrary. By Theorem 7.1, $\bar{f}=h_{1} \cdots h_{k}$ for some $h_{1}, \ldots, h_{k} \in E\left(D_{m}\right)$. By Lemma 6.2, we may choose $e_{1}, \ldots, e_{k} \in E_{a}\left(D_{m}^{a}\right)$ such that $\bar{e}_{i}=h_{i}$ for each $i$. Now put $g=e_{1} \star \cdots \star e_{k} \in\left\langle E_{a}\left(D_{m}^{a}\right)\right\rangle_{a}$. Then $\bar{g}=\bar{f}$, so by Lemma 5.16 , there exist $e_{0}, e_{k+1} \in E_{a}\left(D_{m}^{a}\right)$ such that $f=e_{0} \star g \star e_{k+1} \in\left\langle E_{a}\left(D_{m}^{a}\right)\right\rangle_{a}$.

We now prove the statement about rank and idempotent rank. Note that $I_{1}^{a}=D_{1}^{a}=D_{1}$ is an $n$-element right zero semigroup, so the result is trivial for $m=1$; see also Remark 5.19 . So we assume $1<m<r$ from now on. More generally, if $J$ is a maximal $\mathscr{J}$-class of a finite semigroup $S$, and if the $\mathscr{R}$-classes contained in $J$ are $R_{1}, \ldots, R_{q}$, then any generating set for $S$ must intersect each $R_{p}$ non-trivially (for example, this follows from [42, Exercise 12, p98] or from stability [60, Definition A.2.1]). In particular, it follows from Theorem 5.7 (vi) that $\operatorname{rank}\left(I_{m}^{a}\right) \geq m^{n-r} S(r, m)$. To complete the proof, it suffices to show that there exists $U \subseteq E_{a}\left(D_{m}^{a}\right)$ with $|U|=m^{n-r} S(r, m)$ and such that $\langle U\rangle_{a}$ contains $D_{m}^{a}$. We now construct such a $U$.

First, let $V \subseteq E\left(D_{m}\right) \subseteq \mathcal{T}_{A}$ be such that $|V|=S(r, m)$ and $I_{m}=\langle V\rangle$. Fix some $v \in V$, write $\operatorname{im}(v)=$ $\left\{a_{i_{1}}, \ldots, a_{i_{m}}\right\}$, and put $I=\left\{i_{1}, \ldots, i_{m}\right\}$. Then $H_{v} \phi^{-1}$ is an $\widehat{\mathscr{H}}$-class of $P$, and is an $m^{n-r} \times \Lambda_{I}$ rectangular group. Put $B_{v}=E_{a}\left(H_{v} \phi^{-1}\right)$, so that $B_{v}$ is a $m^{n-r} \times \Lambda_{I}$ rectangular band. Since $m^{n-r} \geq \Lambda_{I}$, we see by Lemma 6.6 that $\operatorname{rank}\left(B_{v}\right)=\operatorname{idrank}\left(B_{v}\right)=m^{n-r}$, so we may choose some $U_{v} \subseteq B_{v}$ with $\left|U_{v}\right|=m^{n-r}$ and $B_{v}=\left\langle U_{v}\right\rangle_{a}$, noting that $\bar{u}=v$ for all $u \in U_{v}$. Note that for any $h \in D_{m}^{a}$ with $\operatorname{ker}(\bar{h})=\operatorname{ker}(v), U_{v}$ contains some $u$ with $\operatorname{ker}(u)=\operatorname{ker}(h)$; a similar statement holds for images. Now put $U=\bigcup_{v \in V} U_{v}$, noting that $U \subseteq E_{a}\left(D_{m}^{a}\right)$ and $|U|=m^{n-r} S(r, m)$. Let $f \in D_{m}^{a}$ be arbitrary, and consider an expression $\bar{f}=v_{1} \cdots v_{k}$, where $v_{1}, \ldots, v_{k} \in V$. Note that, $\operatorname{since} \operatorname{rank}\left(v_{j}\right)=m$ for each $j \in \mathbf{k}$, we have $\operatorname{ker}\left(v_{1}\right)=\operatorname{ker}(\bar{f})$ and $\operatorname{im}\left(v_{k}\right)=\operatorname{im}(\bar{f})$. For each $j \in \mathbf{k}$, we choose some $u_{j} \in U_{v_{j}}$, but we make these choices so that $\operatorname{ker}\left(u_{1}\right)=\operatorname{ker}(f)$ and $\operatorname{im}\left(u_{k}\right)=\operatorname{im}(f)$. Put $g=u_{1} \star \cdots \star u_{k}$. Then $\operatorname{rank}(g)=\operatorname{rank}(\bar{g})=\operatorname{rank}(\bar{f})=\operatorname{rank}(f)$ so, since $\operatorname{rank}\left(u_{j}\right)=\operatorname{rank}\left(\bar{u}_{j}\right)=m$ for each $j$, we see that $\operatorname{ker}(g)=\operatorname{ker}\left(u_{1}\right)=\operatorname{ker}(f)$ and $\operatorname{im}(g)=\operatorname{im}\left(u_{k}\right)=\operatorname{im}(f)$. Together with $\bar{f}=\bar{g}$, this shows that $f=g \in\langle U\rangle_{a}$, and completes the proof. 
Remark 7.5. Again, we note the similarity between Theorem 7.4 and [64, Theorem 4.4], where it is shown that the proper ideals, there denoted $Q(F ; m)$, of $\operatorname{Reg}(\mathcal{T}(X, A))$ are idempotent generated, and have rank and idempotent rank equal to $m^{n-r} S(r, m)$. See also Remark 5.19. We also note that an alternative approach exists to tackle problems such as those we addressed in this section; namely, making use of the general results of Gray [29,30] on (idempotent) rank in completely 0-simple semigroups.

\section{Acknowledgement}

We kindly thank the referee for his/her careful reading of the manuscript and for some helpful suggestions.

\section{References}

[1] A. Ja. Aǔzenštat. Defining relations of finite symmetric semigroups (in Russian). Mat. Sb. N.S., 45 (87):261-280, 1958.

[2] J. Araújo and J. D. Mitchell. Relative ranks in the monoid of endomorphisms of an independence algebra. Monatsh. Math., 151(1):1-10, 2007.

[3] Janet E. Ault. Semigroups with midunits. Semigroup Forum, 6(4):346-351, 1973.

[4] Janet E. Ault. Semigroups with midunits. Trans. Amer. Math. Soc., 190:375-384, 1974.

[5] Gonca Ayık, Hayrullah Ayık, Leyla Bugay, and Osman Kelekci. Generating sets of finite singular transformation semigroups. Semigroup Forum, 86(1):59-66, 2013.

[6] Gonca Ayık, Hayrullah Ayık, and John M. Howie. On factorisations and generators in transformation semigroups. Semigroup Forum, 70(2):225-237, 2005.

[7] Gonca Ayık, Hayrullah Ayık, Yusuf Ünlü, and John M. Howie. Rank properties of the semigroup of singular transformations on a finite set. Comm. Algebra, 36(7):2581-2587, 2008.

[8] T. S. Blyth and J. B. Hickey. RP-dominated regular semigroups. Proc. Roy. Soc. Edinburgh Sect. A, 99(1-2):185-191, 1984.

[9] W. P. Brown. Generalized matrix algebras. Canad. J. Math., 7:188-190, 1955.

[10] Karen Chase. Sandwich semigroups of binary relations. Discrete Math., 28(3):231-236, 1979.

[11] J. Cichoń, J. D. Mitchell, M. Morayne, and Y. Péresse. Relative ranks of Lipschitz mappings on countable discrete metric spaces. Topology Appl., 158(3):412-423, 2011.

[12] Miroslav Ćirić and Stojan Bogdanović. The lattice of varieties of bands. In Semigroups and applications (St. Andrews, 1997), pages 47-61. World Sci. Publ., River Edge, NJ, 1998.

[13] Miroslav Ćirić, Žarko Popović, and Stojan Bogdanović. Effective subdirect decompositions of regular semigroups. Semigroup Forum, 77(3):500-519, 2008.

[14] Igor Dolinka and James East. Semigroups of rectangular matrices under a sandwich operation. Preprint, 2015, arXiv: 1503.03139.

[15] Igor Dolinka and James East. Idempotent generation in the endomorphism monoid of a uniform partition. Comm. Algebra, (to appear), arXiv:1407.3312.

[16] James East. On the singular part of the partition monoid. Internat. J. Algebra Comput., 21(1-2):147-178, 2011.

[17] James East and D. G. FitzGerald. The semigroup generated by the idempotents of a partition monoid. J. Algebra, 372:108-133, 2012

[18] James East and R. D. Gray. Diagram monoids and Graham-Houghton graphs: idempotents and generating sets of ideals. Preprint, 2015, arXiv: 1404.2359.

[19] J. A. Erdos. On products of idempotent matrices. Glasgow Math. J., 8:118-122, 1967.

[20] Isidore Fleischer. A note on subdirect products. Acta Math. Acad. Sci. Hungar., 6:463-465, 1955.

[21] John Fountain. Products of idempotent integer matrices. Math. Proc. Cambridge Philos. Soc., 110(3):431-441, 1991.

[22] John Fountain and Andrew Lewin. Products of idempotent endomorphisms of an independence algebra of finite rank. Proc. Edinburgh Math. Soc. (2), 35(3):493-500, 1992.

[23] John Fountain and Andrew Lewin. Products of idempotent endomorphisms of an independence algebra of infinite rank. Math. Proc. Cambridge Philos. Soc., 114(2):303-319, 1993.

[24] L. Fuchs. On subdirect unions. I. Acta Math. Acad. Sci. Hungar., 3:103-120, 1952.

[25] Olexandr Ganyushkin and Volodymyr Mazorchuk. Classical finite transformation semigroups, an introduction, volume 9 of Algebra and Applications. Springer-Verlag London, Ltd., London, 2009.

[26] The GAP Group. GAP - Groups, Algorithms, and Programming, Version 4.7.8, 2015.

[27] Gracinda Gomes and John M. Howie. On the ranks of certain finite semigroups of transformations. Math. Proc. Cambridge Philos. Soc., 101(3):395-403, 1987.

[28] Gracinda M. S. Gomes and John M. Howie. On the ranks of certain semigroups of order-preserving transformations. Semigroup Forum, 45(3):272-282, 1992.

[29] R. Gray. Idempotent rank in endomorphism monoids of finite independence algebras. Proc. Roy. Soc. Edinburgh Sect. A, 137(2):303-331, 2007.

[30] R. Gray. Hall's condition and idempotent rank of ideals of endomorphism monoids. Proc. Edinb. Math. Soc. (2), 51(1):57$72,2008$.

[31] R. Gray and N. Ruškuc. Generating sets of completely 0-simple semigroups. Comm. Algebra, 33(12):4657-4678, 2005. 
[32] J. B. Hickey. Semigroups under a sandwich operation. Proc. Edinburgh Math. Soc. (2), 26(3):371-382, 1983.

[33] J. B. Hickey. On variants of a semigroup. Bull. Austral. Math. Soc., 34(3):447-459, 1986.

[34] P. M. Higgins, J. M. Howie, J. D. Mitchell, and N. Ruškuc. Countable versus uncountable ranks in infinite semigroups of transformations and relations. Proc. Edinb. Math. Soc. (2), 46(3):531-544, 2003.

[35] P. M. Higgins, J. D. Mitchell, M. Morayne, and N. Ruškuc. Rank properties of endomorphisms of infinite partially ordered sets. Bull. London Math. Soc., 38(2):177-191, 2006.

[36] P. M. Higgins, J. D. Mitchell, and N. Ruškuc. Generating the full transformation semigroup using order preserving mappings. Glasg. Math. J., 45(3):557-566, 2003.

[37] Peter M. Higgins. Techniques of semigroup theory. Oxford Science Publications. The Clarendon Press, Oxford University Press, New York, 1992.

[38] Peter M. Higgins, John M. Howie, and Nikola Ruškuc. Generators and factorisations of transformation semigroups. Proc. Roy. Soc. Edinburgh Sect. A, 128(6):1355-1369, 1998.

[39] J. M. Howie. The subsemigroup generated by the idempotents of a full transformation semigroup. J. London Math. Soc., 41:707-716, 1966.

[40] J. M. Howie. Products of idempotents in certain semigroups of transformations. Proc. Edinburgh Math. Soc. (2), 17:223236, 1970/71.

[41] J. M. Howie. Idempotent generators in finite full transformation semigroups. Proc. Roy. Soc. Edinburgh Sect. A, 81(34):317-323, 1978.

[42] John M. Howie. Fundamentals of semigroup theory, volume 12 of London Mathematical Society Monographs. New Series. The Clarendon Press, Oxford University Press, New York, 1995. Oxford Science Publications.

[43] John M. Howie, Ewing L. Lusk, and Robert B. McFadden. Combinatorial results relating to products of idempotents in finite full transformation semigroups. Proc. Roy. Soc. Edinburgh Sect. A, 115(3-4):289-299, 1990.

[44] John M. Howie and Robert B. McFadden. Idempotent rank in finite full transformation semigroups. Proc. Roy. Soc. Edinburgh Sect. A, 114(3-4):161-167, 1990.

[45] John M. Howie, N. Ruškuc, and P. M. Higgins. On relative ranks of full transformation semigroups. Comm. Algebra, 26(3):733-748, 1998.

[46] Yupaporn Kemprasit. Regularity and unit-regularity of generalized semigroups of linear transformations. Southeast Asian Bull. Math., 25(4):617-622, 2002.

[47] T. A. Khan and M. V. Lawson. Variants of regular semigroups. Semigroup Forum, 62(3):358-374, 2001.

[48] Naoki Kimura. The structure of idempotent semigroups. I. Pacific J. Math., 8:257-275, 1958.

[49] G. M. Kudryavtseva and G. Y. Tsyaputa. The automorphism group of the sandwich inverse symmetric semigroup. Bulletin of the University of Kiev, Series: Mechanics and Mathematics, (13-14):101-105, 2005, available at arXiv:0509678.

[50] E. S. Lyapin. Semigroups (in Russian). Gosudarstv. Izdat. Fiz.-Mat. Lit., Moscow, 1960.

[51] K. D. Magill, Jr. and S. Subbiah. Green's relations for regular elements of sandwich semigroups. I. General results. Proc. London Math. Soc. (3), 31(2):194-210, 1975.

[52] K. D. Magill, Jr. and S. Subbiah. Green's relations for regular elements of sandwich semigroups. II. Semigroups of continuous functions. J. Austral. Math. Soc. Ser. A, 25(1):45-65, 1978.

[53] Kenneth D. Magill, Jr. Semigroup structures for families of functions. I. Some homomorphism theorems. J. Austral. Math. Soc., 7:81-94, 1967.

[54] Victor Maltcev and Volodymyr Mazorchuk. Presentation of the singular part of the Brauer monoid. Math. Bohem., 132(3):297-323, 2007.

[55] V. Mazorchuk and G. Tsyaputa. Isolated subsemigroups in the variants of $\mathcal{T}_{n}$. Acta Math. Univ. Comenian. (N.S.), $77(1): 63-84,2008$.

[56] Suzana Mendes-Gonçalves and Robert P. Sullivan. Semigroups of transformations restricted by an equivalence. Cent. Eur. J. Math., 8(6):1120-1131, 2010.

[57] J. D. Mitchell. The Semigroups package for GAP, Version 2.1. http://tinyurl.com/semigroups, 2014.

[58] Eliakim Hastings Moore. Concerning the abstract groups of order $k$ ! and $\frac{1}{2} k$ ! holohedrically isomorphic with the symmetric and the alternating substitution-groups on $k$ letters. Proc. London Math. Soc., 28(1):357-366, 1897.

[59] K. S. Subramonian Nambooripad. The natural partial order on a regular semigroup. Proc. Edinburgh Math. Soc. (2), 23(3):249-260, 1980.

[60] John Rhodes and Benjamin Steinberg. The q-theory of finite semigroups. Springer Monographs in Mathematics. Springer, New York, 2009.

[61] N. Ruškuc. On the rank of completely 0-simple semigroups. Math. Proc. Cambridge Philos. Soc., 116(2):325-338, 1994.

[62] Jintana Sanwong. The regular part of a semigroup of transformations with restricted range. Semigroup Forum, 83(1):134$146,2011$.

[63] Jintana Sanwong and Worachead Sommanee. Regularity and Green's relations on a semigroup of transformations with restricted range. Int. J. Math. Math. Sci., 2008, Art. ID 794013, 11 pp.

[64] Worachead Sommanee and Jintana Sanwong. Rank and idempotent rank of finite full transformation semigroups with restricted range. Semigroup Forum, 87(1):230-242, 2013.

[65] J. S. V. Symons. On a generalization of the transformation semigroup. J. Austral. Math. Soc., 19:47-61, 1975.

[66] J. S. V. Symons. Some results concerning a transformation semigroup. J. Austral. Math. Soc., 19(4):413-425, 1975.

[67] Gabriel Thierrin. Demi-groupes inversés et rectangulaires (in French). Acad. Roy. Belg. Bull. Cl. Sci. (5), 41:83-92, 1955.

[68] Melvin C. Thornton. Regular elements in sandwich semigroups of binary relations. Discrete Math., 41(3):303-307, 1982.

[69] G. Y. Tsyaputa. Transformation semigroups with the deformed multiplication (in Ukrainian). Bulletin of the University of Kiev, Series: Mechanics and Mathematics, (3):82-88, 2003, English version available at arXiv:0508386.

[70] G. Y. Tsyaputa. Green's relations on the deformed transformation semigroups. Algebra Discrete Math., (1):121-131, 2004. 
[71] G. Y. Tsyaputa. Deformed multiplication in the semigroup $\mathcal{P} \mathcal{T}_{n}$ (in Ukrainian). Bulletin of the University of Kiev, Series: Mechanics and Mathematics, (11-12):35-38, 2004, English version available at arXiv:0508623.

[72] Galyna Tsyaputa. Isolated and nilpotent subsemigroups in the variants of $\mathcal{I S}_{n}$. Algebra Discrete Math., (1):89-97, 2006.

[73] N. N. Vorob'ev. On symmetric associative systems. Leningrad. Gos. Ped. Inst. Uč. Zap., 89:161-166, 1953.

[74] E. M. Wright. The number of irreducible tournaments. Glasgow Math. J., 11:97-101, 1970.

[75] Miyuki Yamada. A note on middle unitary semigroups. Kōdai Math. Sem. Rep., 7:49-52, 1955. 